\title{
“Minimal Flavour Violation” for leptoquarks
}

\author{
Sacha Davidson ${ }^{a}$ and Sébastien Descotes-Genon ${ }^{b}$ \\ ${ }^{a} I P N L$, Université de Lyon, Université Lyon 1, CNRS/IN2P3, \\ 4 rue E. Fermi 69622 Villeurbanne cedex, France \\ ${ }^{b}$ Laboratoire de Physique Théorique, CNRS/Univ. Paris-Sud 11 (UM8627), \\ 91405 Orsay Cedex, France \\ E-mail: s.davidson@ipnl.in2p3.fr, descotes@th.u-psud.fr
}

ABSTRACT: Scalar leptoquarks, with baryon and lepton number conserving interactions, could have $\mathrm{TeV}$ scale masses, and be produced at colliders or contribute to a wide variety of rare decays. In pursuit of some insight as to the most sensitive search channels, we assume that the leptoquark-lepton-quark coupling can be constructed from the known mass matrices. We estimate the rates for selected rare processes in three cases: leptoquarks carrying lepton and quark flavour, leptoquarks with quark flavour only, and unflavoured leptoquarks. We find that leptoquark decay to top quarks is an interesting search channel.

KeYwords: Beyond Standard Model, Rare Decays

ARXiv EPRINT: 1009.1998 


\section{Contents}

1 Introduction 1

2 Notation 3

2.1 Flavour symmetries and symmetry breaking terms 3

2.2 Leptoquarks 4

$\begin{array}{lll}3 & \text { Flavour singlet leptoquarks } & 7\end{array}$

$\begin{array}{ll}3.1 \text { Adding a new spurion } & 7\end{array}$

$\begin{array}{lll}3.2 & \text { Some comments } & 10\end{array}$

4 Leptoquarks with quark flavour $\quad 13$

$\begin{array}{ll}4.1 \text { Maximal coupling } & 14\end{array}$

$\begin{array}{lll}4.2 & \text { Coherent gauge and flavour assignments } & 16\end{array}$

$\begin{array}{lll}5 & \text { Leptoquarks with quark and lepton flavour } & 19\end{array}$

$\begin{array}{lll}6 & \text { Summary and discussion } & 22\end{array}$

\section{Introduction}

Like Higgs bosons, scalar leptoquarks $S$ have renormalisable couplings $\lambda^{\mathrm{LQ}}$ to two fermions. One could anticipate that the flavour structure of the leptoquark and of the Yukawa couplings has the same origin, suggesting that, from a phenomenological bottom-up perspective, the $\lambda^{\mathrm{LQ}}$ might be constructible out of the Standard Model (SM) Yukawa matrices $Y_{f}$. We explore various possibilities in this paper. Since our building blocks are the known mass matrices, we call this "Minimal Flavour Violation (MFV) for leptoquarks".

Leptoquarks ${ }^{1}$ do not address topical open questions such as the identity of dark matter or the origin of the electroweak scale. However, they have some motivation. The SM contains bosons with either colour (gluons) or charge (Higgs and SU(2) gauge bosons), but no coloured and charged bosons which could have renormalisable interactions with a lepton and a quark (leptoquarks). Nonetheless, anomaly cancellation implies that the quark and lepton sectors are related. Theories (GUTs) that unify the strong and electroweak interactions frequently have B and L violating leptoquark gauge bosons, whose values are kept at the GUT scale because they could mediate proton decay [3]. In this paper, we are interested in B and L conserving scalar leptoquarks, with LHC-accessible masses. They could arise in technicolour models [4], in R-parity violating Supersymmetry (see e.g. [5]), or be low-energy remnants of a GUT $[6,7]$. From a more phenomenological perspective,

\footnotetext{
${ }^{1}$ For a review see e.g. the following and references and citations thereof $[1,2]$.
} 
their strong interactions make them interesting for hadron colliders, and their lepton-quark couplings can be probed in rare decay experiments. The prospects for detecting leptoquarks at the LHC have been discussed in various models [8-11].

Some insight into "where to look for leptoquarks", would be useful. For instance, are B decays, involving third generation fermions, a more promising place to look for leptoquarks than the more sensitive Kaon decays? And how do they compare with $t \bar{t}$ production at hadron colliders ? To address such questions requires some knowledge about the leptoquark masses $m_{S}$ and couplings $\lambda^{\mathrm{LQ}}$. From a phenomenological perspective, the first solid step is to extract from current rare process data the bounds on $\left|\lambda^{\mathrm{LQ}}\right|^{2} / m_{S}^{2}$, as was done recently, for instance, in $[12]^{2}$ and $[13,14]$. In this paper, we fix $m_{S} \sim 300 \mathrm{GeV}$, which is of order of the Tevatron lower limits ${ }^{3}[15-18]$

$$
m_{S} \gtrsim 250-300 \mathrm{GeV}
$$

and accessible to the LHC [19-22]. Then we explore various patterns for the $\lambda^{\mathrm{LQ}}$, constructed from the SM Yukawa couplings, which are consistent with the bounds. The patterns give predictions for the most promising search channels for leptoquarks.

To combine SM Yukawa couplings into a leptoquark coupling is not immediately obvious, because the quark Yukawa couplings connect the quark flavour spaces, and $Y_{e}$ connects the lepton flavour spaces:

$$
\begin{aligned}
& u_{R} \leftarrow Y_{u} \rightarrow q \leftarrow Y_{d} \rightarrow d_{R} \\
& \ell \leftarrow Y_{e} \rightarrow e_{R}
\end{aligned}
$$

but there is no bridge between leptons and quarks. We consider three independent ways to construct a lepton-quark-leptoquark interaction, which differ by the quark- and leptonflavour assigned to the leptoquarks:

(1) flavour-singlet leptoquarks;

(2) leptoquarks with non-trivial transformation properties only under the quark-flavour groups;

(3) leptoquarks with both quark and lepton flavour indices.

In the first case, we are forced to introduce a new flavour-breaking structure connecting the quark and lepton flavour spaces. In the second, following [23], we can build flavourinvariant operators using the majorana neutrino mass matrix, and in the third case, we need only the SM Yukawa couplings as symmetry breaking structures. In all cases, there are a number of options, so we make additional assumptions, aimed to maximise the leptoquark coupling $\lambda^{\mathrm{LQ}}$.

\footnotetext{
${ }^{2}$ Some errors in the arXiv version are corrected in the journal.

${ }^{3}$ The Tevatron bounds vary depending on the final state fermions and assumed Branching Ratios.
} 


\section{Notation}

\subsection{Flavour symmetries and symmetry breaking terms}

The kinetic terms of three generations of Standard Model (SM) fermions have a global $\mathrm{U}(3)_{q} \times \mathrm{U}(3)_{u} \times \mathrm{U}(3)_{d} \times \mathrm{U}(3)_{\ell} \times \mathrm{U}(3)_{e}$ symmetry, which is broken by the quark and charged lepton Yukawa couplings to $\mathrm{U}(1)_{Y} \times \mathrm{U}(1)_{B} \times \mathrm{U}(1)_{L e} \times \mathrm{U}(1)_{L \mu} \times \mathrm{U}(1)_{L \tau}$ (where $\mathrm{U}(1)_{Y}$ is global hypercharge, $\mathrm{U}(1)_{B}$ is baryon number, and the $\mathrm{U}(1)_{L i}$ are lepton flavours). Majorana neutrino masses ${ }^{4}$ will be included in section 4. As is well known, the Yukawa couplings give hierarchical masses to the charged leptons and quarks, and identify a unique "mass eigenstate basis" in the flavour spaces of the $u, d, e$ and $\ell$. In the flavour space of the $q \mathrm{~s}$, there are the two mass eigenstate bases of the $d_{L} \mathrm{~s}$ and the $u_{L} \mathrm{~s}$, which are related by the CKM matrix $K$.

There is a large body of precise flavour data in the quark sector, which agrees with SM expectations. This implies that any flavoured interactions of new particles accessible to the LHC, should, somehow, "align" themselves on the eigenbases of the quark Yukawa couplings and share their eigenvalues. This is elegantly obtained with the Minimal Flavour Violation (MFV) hypothesis [24-26]: only the Yukawa matrices can break the global U $(3)^{5}$. Extending the MFV framework to sectors other than the quarks has been previously considered by various authors [27-31].

We write the SM Yukawa matrices with the flavour indices ordered doublet-singlet. For instance, in the case of the charged leptons:

$$
\left[Y_{e}\right]^{I n}\langle H\rangle \bar{e}_{n} \ell_{I}+\text { h.c. } \supset m_{e} \overline{e_{R}} e_{L}+\text { h.c. }
$$

where $\langle H\rangle=v=175 \mathrm{GeV}$ and the diagonal Yukawa matrix of fermion $f$ will be denoted $D_{f}$. Capitalised roman indices $I, J, K$ correspond to $\mathrm{SU}(2)$ doublets $q, \ell$, lower case roman indices $(i, j, k)$ are carried by the singlets: $e, u, d$. Preference is given to the beginning of the alphabet for leptons, and the later part for quarks. Chiral subscripts $L, R$ are suppressed to avoid confusion with flavour indices.

We take the perspective that the largest eigenvalue of the $\left[Y_{f}\right]$ may be $\mathcal{O}(1)$. We implement this by considering a Higgs sector of two doublets, $H_{u}$ and $H_{d}$, coupled separately to up-type quarks $\left(H_{u}\right)$ and down-type quarks and charged leptons $\left(H_{d}\right)$. This possibility allows us to change the relative normalization of the Yukawa couplings, changing the ratio of the two Higgs vacuum expectation values: $\tan \beta=\left\langle H_{u}\right\rangle /\left\langle H_{d}\right\rangle$. In particular, for $\tan \beta \gg 1$, we have:

$$
\begin{aligned}
& D_{e} \equiv \operatorname{diag}\left\{y_{e}, y_{\mu}, y_{\tau}\right\}=\frac{\tan \beta}{v} \operatorname{diag}\left\{m_{e}, m_{\mu}, m_{\tau}\right\}, \\
& D_{d}=\frac{\tan \beta}{v} \operatorname{diag}\left\{m_{d}, m_{s}, m_{d}\right\}, \quad D_{u}=\frac{1}{v} \operatorname{diag}\left\{m_{u}, m_{c}, m_{t}\right\},
\end{aligned}
$$

and the one-Higgs doublet case is recovered for $\tan \beta=1$. Given the misalignment of the two quark Yukawa couplings is not affected by their overall normalization, present flavour

\footnotetext{
${ }^{4}$ Dirac neutrino masses are beyond the scope of this preliminary analysis. They allow additional leptoquarks, interacting with the singlet $\nu_{R} \mathrm{~s}$. But larger quark and lepton flavour-changing rates might be obtained in this case.
} 
data are compatible with large $\tan \beta$ values. The latter choice is particularly interesting since it could allow to consider a scenario where top, bottom and tau Yukawa couplings are of order 1 .

For further convenience, we define the following combinations of SM Yukawa matrices:

$W_{e}=Y_{e} Y_{e}^{\dagger}, \quad W_{d}=Y_{d} Y_{d}^{\dagger}, \quad W_{u}=Y_{u} Y_{u}^{\dagger}, \quad \widetilde{W}_{e}=Y_{e}^{\dagger} Y_{e}, \quad \widetilde{W}_{d}=Y_{d}^{\dagger} Y_{d}, \quad \widetilde{W}_{u}=Y_{u}^{\dagger} Y_{u}$

In section 4, we will include neutrino masses, assumed to be majorana, with a mass matrix $\left[m_{\nu}\right]$ included in the Lagrangian as

$$
\frac{\left[m_{\nu}\right]_{I J}}{2} \bar{\nu}^{c}{ }_{I} \nu_{J}+\text { h.c. }
$$

\subsection{Leptoquarks}

We consider $\mathrm{SU}(2)$ singlet and doublet scalar leptoquarks, with renormalisable $B$ and $L$ conserving interactions. In the notation of Buchmuller,Ruckl and Wyler [32], ${ }^{5}$ these can be added to the SM Lagrangian as:

$$
\begin{aligned}
\mathcal{L}_{\mathrm{LQ}}= & S_{0}\left(\lambda_{\mathbf{L S}_{\mathbf{0}}} \bar{\ell} i \tau_{2} q^{c}+\lambda_{\mathbf{R S}_{\mathbf{0}}} \bar{e} u^{c}\right)+\tilde{S}_{0} \tilde{\lambda}_{\mathbf{R} \tilde{\mathbf{S}}_{\mathbf{0}}} \bar{e} d^{c} \\
& +\left(\lambda_{\mathbf{L S}_{\mathbf{2}}} \bar{\ell} u+\lambda_{\mathbf{R S}_{\mathbf{2}}} \bar{e} q\left[i \tau_{2}\right]\right) S_{2}+\tilde{\lambda}_{\mathbf{L} \tilde{\mathbf{S}}_{\mathbf{2}}} \bar{\ell} d \tilde{S}_{2}+\text { h.c. }
\end{aligned}
$$

where the $\lambda$ s are $3 \times 3$ matrices with index order lepton-quark, and $\tau_{2}$ is a Pauli matrix, so $i \tau_{2}$ provides the antisymmetric $\mathrm{SU}(2)$ contraction. Notice that $\left(\overline{q^{c}}{ }_{P} i \tau_{2} \ell_{I}\right) \sim u_{P} e_{I}-d_{P} \nu_{I}$, so the leptoquark $S_{0}$ does not interact with $d e$, and cannot mediate processes such as $K_{L} \rightarrow \mu e$ at tree level (see table 1). In this Lagrangian, the leptoquark leaves the vertex into which enter the leptons. So for instance, the SU(2) singlets leptoquarks have fermion number 2 , the doublets carry no fermion number. Other quantum numbers are listed below $\left(Q_{\mathrm{em}}=Y / 2+T_{3}, T_{3}= \pm 1 / 2\right.$ for doublets $)$

$\begin{array}{lccccc}\text { leptoquark } & Y & B & L & \mathrm{SU}(2) & \text { couplings } \\ S_{0} & -2 / 3 & 1 / 3 & 1 & 1 & \lambda_{\mathbf{L S}}, \lambda_{\mathbf{R S}} \\ \tilde{S}_{0} & -8 / 3 & 1 / 3 & 1 & 1 & \tilde{\lambda}_{\mathbf{R}} \tilde{\mathbf{S}}_{\mathbf{0}} \\ S_{2} & -7 / 3 & -1 / 3 & 1 & 2 & \lambda_{\mathbf{L S}}, \lambda_{\mathbf{R S}_{\mathbf{2}}} \\ \tilde{S}_{2} & -1 / 3 & -1 / 3 & 1 & 2 & \tilde{\lambda}_{\mathbf{L}} \tilde{\mathbf{S}}_{\mathbf{2}}\end{array}$

In addition to the Tevatron lower bound on leptoquark masses given in eq. (1.1), there is a lower bound from HERA [33-35] $m_{S}>250-300 \mathrm{GeV}$ (for leptoquarks coupling to firstgeneration fermions with $\lambda \sim 0.1$ ) and a variety of constraints from low energy/precision experiments which are sensitive to the coefficients of dimension six operators mediated by leptoquarks. Such operators include the quark and charged lepton dipoles and four fermion operators involving a quark, an anti-quark, a lepton and an anti-lepton (which we refer to as two-quark-two-lepton operators). The four-fermion operators can be Fierz-rearranged to

\footnotetext{
${ }^{5}$ We took the complex conjugate of $\mathcal{L}$, to obtain fermion field order lepton-quark, without taking the hermitian conjugate of the $\lambda \mathrm{s}$. So our $\lambda \mathrm{s}$ are $\lambda_{\mathrm{BRW}}^{*}$.
} 
(lepton-current) $\times$ (quark current) form (see table 1 ) which is more convenient for comparing to SM processes. Following [12], ${ }^{2}$ the coefficients of these $V \pm A$ two-quark-two-lepton operators can be normalised as

$$
\frac{C_{X}^{i j r s}}{m_{S}^{2}}=\varepsilon_{X}^{i j r s} \frac{4 G_{F}}{\sqrt{2}}
$$

and experimental constraints can be set on these four-index $\varepsilon^{i j p q} \mathrm{~s}$.We use the recent bounds of [14], which arise from leptonic and semi-leptonic decays of pseudoscalar mesons (e.g. $R_{K} \equiv \Gamma\left(K^{+} \rightarrow e^{+} \nu\right) / \Gamma\left(K^{+} \rightarrow \mu^{+} \nu\right)$ and $\left.K^{+} \rightarrow \pi^{+} \nu \bar{\nu}\right)$, flavour-changing but generationdiagonal meson decays such as $K_{L} \rightarrow \mu^{ \pm} e^{\mp}$, contact interaction searches at colliders and $\mu-e$ conversion on nuclei (we neglect leptoquark loop contributions to four-quark operators, which are constrained by meson-anti-meson mixing [13]). Considering absolute values only and assuming $m_{S} \sim 300 \mathrm{GeV}$, the upper bounds of [14] on the $\varepsilon$ s imply that

$$
\frac{\lambda^{2}}{6} \lesssim \varepsilon
$$

for appropriate indices.

At dimension six, there are also flavour changing dipole operators involving two fermions and a gauge boson $(\gamma, Z, g)$, which can contribute to anomalous $Z$ decays [36, 37] and processes such as $b \rightarrow s \gamma$ and $\mu \rightarrow e \gamma$. After Spontaneous Symmetry Breaking, the dipole that mediates $f_{2} \rightarrow f_{1} \gamma$ can be written as $[12]^{2}$

$\frac{e_{e m} m_{2}}{2} \bar{f}_{1} \sigma^{\alpha \beta}\left(A_{L} P_{L}+A_{R} P_{R}\right) f_{2} F_{\alpha \beta}+h . c . \equiv \frac{e_{e m} m_{2} G_{F}}{2} \bar{f}_{1} \sigma^{\alpha \beta}\left(\varepsilon_{L}^{f_{1} f_{2}} P_{L}+\varepsilon_{R}^{f_{1} f_{2}} P_{R}\right) f_{2} F_{\alpha \beta}+$ h.c.

where the second equality defines the dimensionless (two-index) $\varepsilon$ s for these operators. Notice that these effective couplings are defined factorising the heavy fermion mass, as expected from a chirality flip on the external leg. They can be bounded from the experimental limits on the branching ratio, for instance as

$$
B R(\mu \rightarrow e \gamma)=\frac{48 \pi^{3} \alpha}{G_{F}^{2}}\left(A_{L}^{2}+A_{R}^{2}\right)=48 \pi^{3} \alpha\left(\left|\varepsilon_{L}^{e \mu}\right|^{2}+\left|\varepsilon_{R}^{e \mu}\right|^{2}\right)
$$

A list of experimental bounds on selected $\varepsilon$ s, relevant to the scenarios we consider, can be found in tables 2,3 and 4 .

We estimate $\varepsilon^{f_{2} f_{1}}$, arising from the diagrams of figure 1 and of wave function renormalisation, as [38]

$$
\varepsilon^{f_{2} f_{1}}=\frac{1}{96 \pi^{2}} \times\left\{\begin{array}{l}
\lambda_{X} \lambda_{X}^{*}\left(Q_{F}+Q_{S} / 2\right) \\
\frac{m_{F}}{m_{1}} \lambda_{L} \lambda_{R}^{*}\left(\left[9+6 \ln \left(\frac{m_{F}^{2}}{m_{S}^{2}}\right)\right] Q_{F}-3 Q_{S}\right)
\end{array}\right.
$$

where $X=L$ or $R$, and the sign of the electric charges $Q$ is given by the line directions of figure 1. These estimates apply to the case $m_{F} \rightarrow 0$; for a top quark in the loop, the numerical factor is a bit smaller [38].

In principle, additional constraints on leptoquarks could be obtained from electroweak precision observables (such as the oblique parameters $S, T, U \ldots$ ). However, by construction, the oblique parameters are sensitive to the breaking of the SM gauge symmetry (the 


\begin{tabular}{|c|c|c|}
\hline interaction & 4 - fermion vertex & Fierz - transformed vertex \\
\hline$\left(\lambda_{L S_{0}} \overline{q^{c}} i \sigma_{2} \ell+\lambda_{R S_{0}} \overline{u^{c}} e\right) S_{0}^{\dagger}$ & $\begin{array}{l}\frac{\lambda_{R S_{0} \lambda_{R S_{0}}^{*}}}{m_{S}^{2}}\left(\overline{u^{c}} e\right)\left(\bar{e} u^{c}\right) \\
\frac{\lambda_{L S_{0}} \lambda_{L S_{0}}^{*}}{m_{S}^{2}}\left(\bar{q}^{c} i \sigma_{2} \ell\right)\left(\bar{\ell} i \sigma_{2} q^{c}\right) \\
\frac{\lambda_{R S_{0}} \lambda_{L S_{0}}^{*}}{m_{o}^{2}}\left(\bar{q}^{c} i \sigma_{2} \ell\right)\left(\bar{e} u^{c}\right)\end{array}$ & $\begin{array}{l}\frac{\lambda_{R S_{0}} \lambda_{R S_{0}}^{*}}{2 m_{S}^{2}}\left(\bar{u} \gamma^{\mu} P_{R} u\right)\left(\bar{e} \gamma_{\mu} P_{R} e\right) \\
\frac{\lambda_{L S_{0}} \lambda_{L S_{0}}}{2 m_{S}^{2}}\left(\bar{u} \gamma^{\mu} P_{L} u\right)\left(\bar{e} \gamma_{\mu} P_{L} e\right) \\
\frac{\lambda_{L S_{0}} \lambda_{L S_{0}}}{2 m_{S}^{2}}\left(\bar{d} \gamma^{\mu} P_{L} u\right)\left(\bar{\nu} \gamma_{\mu} P_{L} e\right) \\
\frac{\lambda_{L S_{0}} \lambda_{L S_{0}}}{2 m_{S}^{2}}\left(\bar{d} \gamma^{\mu} P_{L} d\right)\left(\bar{\nu} \gamma_{\mu} P_{L} \nu\right) \\
\frac{\lambda_{R S_{0}} \lambda_{L S_{0}}}{2 m_{S}^{2}}\left(\bar{u} P_{L} u\right)\left(\bar{e} P_{L} e\right)+\cdots \\
\frac{\lambda_{R S_{0}} \lambda_{L S_{0}}}{2 m_{S}^{2}}\left(\bar{u} P_{L} d\right)\left(\bar{e} P_{L} \nu\right)+\cdots\end{array}$ \\
\hline$\lambda_{R \tilde{S}_{0}} \overline{d^{c}} e \tilde{S}_{0}^{\dagger}$ & $\frac{\lambda_{R \tilde{S_{0}}} \lambda_{R \tilde{S_{0}}}^{*}}{m_{S}^{2}}\left(\overline{d^{c}} e\right)\left(\bar{e} d^{c}\right)$ & $\frac{\lambda_{R \tilde{S_{0}}} \lambda_{R \tilde{S_{0}}}^{*}}{2 m_{S}^{2}}\left(\bar{d} \gamma^{\mu} P_{R} d\right)\left(\bar{e} \gamma_{\mu} P_{R} e\right)$ \\
\hline$\left(\lambda_{L S_{2}} \bar{u} \ell+\lambda_{R S_{2}} \bar{q} i \sigma_{2} e\right) S_{2}^{\dagger}$ & $\begin{array}{l}\frac{\lambda_{L S_{2}} \lambda_{L S_{2}}^{*}}{m_{S}^{2}}(\bar{u} \ell)(\bar{\ell} u) \\
\frac{\lambda_{R S_{2}} \lambda_{R S_{2}}^{*}}{m_{S}^{2}}(\bar{q} e)(\bar{e} q) \\
\frac{\lambda_{L S_{2}} \lambda_{R S_{2}}^{*}}{m_{S}^{2}}(\bar{u} \ell)(\bar{e} q)\end{array}$ & $\begin{array}{l}-\frac{\lambda_{L S_{2}} \lambda_{L S_{2}}^{*}}{2 m_{S}^{2}}\left(\bar{u} \gamma^{\mu} P_{R} u\right)\left(\bar{\nu} \gamma_{\mu} P_{L} \nu\right) \\
-\frac{\lambda_{L S_{2}} \lambda_{L S_{2}}^{*}}{2 m_{S}^{2}}\left(\bar{u} \gamma^{\mu} P_{R} u\right)\left(\bar{e} \gamma_{\mu} P_{L} e\right) \\
-\frac{\lambda_{R S_{2}} \lambda_{R S_{2}}^{*}}{2 m_{S}^{2}}\left(\bar{u} \gamma^{\mu} P_{L} u\right)\left(\bar{e} \gamma_{\mu} P_{R} e\right) \\
-\frac{\lambda_{R S_{2} \lambda_{R S_{2}}}}{2 m_{S}^{2}}\left(\bar{d} L \gamma^{\mu} P_{L} d\right)\left(\bar{e} \gamma_{\mu} P_{R} e\right) \\
-\frac{\lambda_{L S_{2}} \lambda_{R S_{2}}^{*}}{2 m_{S S}^{2}}\left(\bar{u} P_{L} d\right)\left(\bar{e} P_{L} \nu\right)+\cdots \\
-\frac{\lambda_{L S_{2}} \lambda_{R S_{2}}^{*}}{2 m_{S}^{2}}\left(\bar{u} P_{L} u\right)\left(\bar{e} P_{L} e\right)+\cdots\end{array}$ \\
\hline$\lambda_{L \tilde{S}_{2}} \bar{d} \ell \tilde{S}_{2}^{\dagger}$ & $\frac{\lambda_{L \tilde{S}_{2} \lambda_{L \tilde{S}_{2}}^{*}}}{m_{S}{ }^{2}}(\bar{d} \ell)(\bar{\ell} d)$ & $\begin{array}{l}-\frac{\lambda_{L \tilde{S}_{2}} \lambda_{L \tilde{S}_{2}}^{*}}{2 \tilde{m}_{S}^{2}}\left(\bar{d} \gamma^{\mu} P_{R} d\right)\left(\bar{\nu} \gamma_{\mu} P_{L} \nu\right) \\
-\frac{\lambda_{L \tilde{S}_{2}} \lambda_{L \tilde{S}_{2}}^{*}}{2 \tilde{m S}^{2}}\left(\bar{d} \gamma^{\mu} P_{R} d\right)\left(\bar{e} \gamma_{\mu} P_{L} e\right)\end{array}$ \\
\hline
\end{tabular}

Table 1. Four fermion operators induced by leptoquarks. After Fierz rearrangement, the effective interactions $\propto \lambda_{L} \lambda_{R}$ also have tensor components, represented as $+\cdots$, which we neglect.

relevant non-renormalisable operators contain the Higgs field), and the Higgs-leptoquark couplings [39] do not concern our analysis. Therefore we expect electroweak precision contraints on the flavoured leptoquark couplings to be unimportant. To check this, one can estimate $[40-42]$

$$
\begin{aligned}
S & \left.\equiv \frac{16 \pi^{2} c_{W}}{g^{2} s_{W}} \frac{d}{d q^{2}} \Pi_{W_{3} B}\left(q^{2}\right)\right|_{q^{2}=0} \simeq-N_{S} N_{c} \frac{Y}{6 \pi} \frac{\Delta^{2}}{m_{S}^{2}} \\
T & \equiv \frac{1}{\alpha}\left(\frac{\Pi_{W W}(0)}{m_{W}^{2}}-\frac{\Pi_{Z Z}(0)}{m_{Z}^{2}}\right) \simeq \frac{N_{S} N_{c}}{16 \pi s_{W}^{2} m_{W}^{2}} \Delta^{2}
\end{aligned}
$$

where $\Delta^{2}$ can be the mass-squared splitting in a doublet, or the singlet-doublet mixing mass $m_{S} \Delta S_{0} \tilde{S}_{2}$ [43], and $N_{S}$ is the number of copies of the leptoquark: three if the leptoquark has quark flavour, nine if it has quark and lepton flavours. Requiring $0 \lesssim T-S \lesssim .1,-.25 \lesssim S+T \lesssim .25^{6}$ suggests that

$$
N_{S} \frac{\Delta^{2}}{m_{S}^{2}} \ll 1
$$

\footnotetext{
${ }^{6}$ For numerical values of masses mass differences and mixing angles see [44].
} 

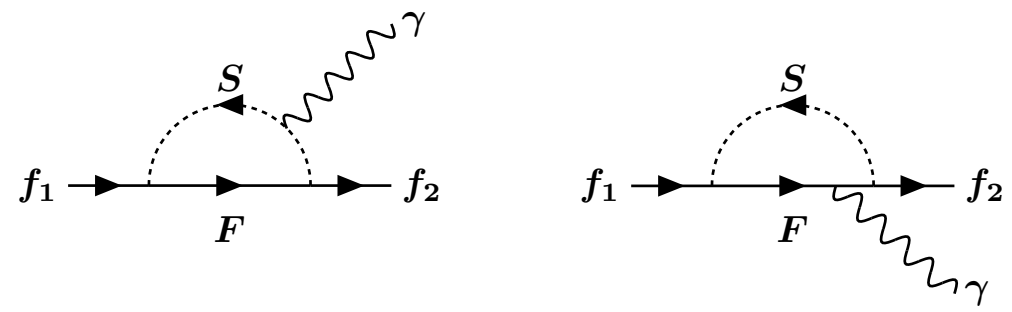

Figure 1. One-loop diagrams mediated by a leptoquark $S$ that could induce $f_{1} \rightarrow f_{2} \gamma$.

is acceptable. A Higgs-leptoquark coupling will neccessarily arise in our models at one loop. If the mass splitting is induced by a third generation fermion loop, with the Higgs boson interacting with the $t$ quark, then $\Delta^{2} \lesssim v^{2} / 16 \pi$, eq. (2.14) is satisfied even for 9 leptoquark flavours, and we conclude the oblique parameters do not provide significant constraints on the masses and flavoured couplings of leptoquarks.

\section{Flavour singlet leptoquarks}

Ideally, we would like to construct, out of SM Yukawa matrices, a leptoquark coupling matrix $[\lambda]$, with one quark flavour index and one lepton flavour index. To see why this is not possible, recall the popular formulation of MFV [26], which identifies the Yukawa matrices as auxiliary fields, or "spurions", whose transformations under the global U $(3)^{5}$ are chosen to ensure the invariance of the Yukawa interactions. Then the spurions get "vacuum expectation values", and the $\mathrm{U}(3)^{5}$ is "spontaneously broken", leaving baryon and lepton number as global symmetries. That is, Lorentz scalars constructed out of SM fermions and spurions do not transform under $B$ or $L$. However, since the leptoquarks carry baryon and lepton number, $[\lambda]^{\mathrm{LQ}}$ (where $L$ and $Q$ are SM lepton and quark fields) cannot be constructed out of the SM spurions. So in this section we consider adding a new spurion.

\subsection{Adding a new spurion}

This new interaction should depart as little as possible from SM flavour structures, and should be consistent with current bounds. We therefore make three assumptions. First, we connect the $u$ singlets to leptons, because constraints on new interactions involving $u, c, t$ quarks are weaker than those involving $d$-type quarks. Secondly, the spurion is taken to be proportional to the unit matrix $\mathcal{I}$, because it only breaks the $\mathrm{SU}(3)_{l} \times \mathrm{SU}(3)_{u}$ symmetry of the kinetic terms to $\mathrm{SU}(3)_{l+u}$ (where $l=\ell$ or $e$ ). This suggests two possible couplings:

$$
\begin{aligned}
& {\left[\lambda_{\mathbf{R S}_{\mathbf{0}}}\right]^{i p} S_{0} \bar{e}_{i} u_{p}^{c}=\lambda_{S_{0}}[\mathcal{I}]^{i p} S_{0} \bar{e}_{i} u_{p}^{c}} \\
& {\left[\lambda_{\mathbf{L S}_{\mathbf{2}}}\right]^{I p} \bar{\ell}_{I} u_{p} S_{2}=\lambda_{S_{\mathbf{2}}}[\mathcal{I}]^{I p} \bar{\ell}_{I} u_{p} S_{2}}
\end{aligned}
$$

where the $\lambda_{S_{0}}$ and $\lambda_{S_{2}}$ are constants not matrices. If $\left[\lambda_{\mathbf{R S}}\right] \propto \mathcal{I}$ in generic bases for the $u$ and $e$ flavour spaces, then it would become a unitary matrix in the mass eigenstate bases of $e$ and $u$. This brings us to our third assumption: we impose that the unit matrix is in the mass bases(because otherwise there are severe constraints from $\mu N \rightarrow e N$ and $\tau \rightarrow \pi^{0} \ell$ ). 
With these assumptions, the most restrictive experimental bound comes from Tevatron searches for contact interactions of the form $\left(\bar{u} \gamma P_{R} u\right)\left(\bar{e} \gamma P_{L, R} e\right)$, and gives $\varepsilon_{\mathrm{eu}}^{1111}<10^{-2}$ and $\varepsilon_{\ell u}^{1111}<1.4 \times 10^{-2}$. These would be satisfied, for $m_{S} \sim 300 \mathrm{GeV}$, by $\lambda_{S_{0}}, \lambda_{S_{2}} \lesssim 1 / 4$.

From the new spurion of eq. (3.1), we can construct couplings of leptoquarks carrying two units of fermion number, to other types of leptons or quarks by multiplying by the SM Yukawas:

$$
\begin{aligned}
{\left[\lambda_{\mathbf{L} \mathbf{S}_{\mathbf{o}}}\right] S_{0} \bar{\ell} i \tau_{2} q^{c} } & =\left[\mathbf{Y}_{\mathbf{e}}^{*} \mathcal{I} \mathbf{Y}_{\mathbf{u}}^{\dagger}\right]^{I P} S_{0} \bar{\ell}_{I} i \tau_{2} q_{P}^{c} \\
{\left[\tilde{\lambda}_{\mathbf{R} \tilde{\mathbf{S}}_{0}}\right] \tilde{S}_{0} \bar{e} d^{c} } & =\left[\mathcal{I} \mathbf{Y}_{\mathbf{u}}^{\dagger} \mathbf{Y}_{\mathbf{d}}\right]^{i p} \tilde{S}_{0} \bar{e}_{i} d_{p}^{c}
\end{aligned}
$$

We have not included $\lambda_{S_{0}}$ in the definition of these other couplings, because this allows them to be larger, and because such an overall scaling of flavoured interactions may be due to some unflavoured physics. So we identify as our new "spurion" the unit matrix $\mathcal{I}$, rather than $\lambda_{S_{0}} \mathcal{I}$ or $\lambda_{S_{2}} \mathcal{I}$. See also the comments in section 3.2.

Similarly, from the new spurion of eq. (3.2), we can construct couplings of the leptoquarks with zero fermion number:

$$
\begin{aligned}
{\left[\lambda_{\mathbf{R S}_{\mathbf{2}}}\right] \bar{e}\left[i \tau_{2} q\right]^{T} S_{2} } & =\left[\mathbf{Y}_{\mathbf{e}}^{\mathbf{T}} \mathcal{I} \mathbf{Y}_{\mathbf{u}}^{\mathbf{T}}\right]^{i P} \bar{e}_{i}\left[i \tau_{2} q_{P}\right]^{T} S_{2} \\
{\left[\tilde{\lambda}_{\mathbf{L} \tilde{\mathbf{S}}_{\mathbf{2}}}\right]^{I p} \bar{\ell}_{I} d_{p} \tilde{S}_{2} } & =\left[\mathcal{I} \mathbf{Y}_{\mathbf{u}}^{\mathbf{T}} \mathbf{Y}_{\mathbf{d}}^{*}\right]^{I p} \bar{\ell}_{I} d_{p} \tilde{S}_{2}
\end{aligned}
$$

The four fermion operators induced respectively by $S_{0}, \tilde{S}_{0}, S_{2}$, and $\tilde{S}_{2}$, and their coefficients, are:

$$
\begin{array}{lll}
\frac{\left|\lambda_{S_{0}}\right|^{2}}{m_{S}^{2}}\left(\bar{u}_{i}^{c} e_{i}\right)\left(\bar{e}_{j} u_{j}^{c}\right), \quad & {\left[\mathbf{D}_{\mathbf{e}} \mathbf{Y}_{\mathbf{u}}^{\mathbf{T}}\right]^{I P} \frac{\left|\lambda_{S_{0}}\right|}{m_{S}^{2}}\left(\bar{q}^{c} P^{i} \tau_{2} \ell_{I}\right)\left(\bar{e}_{j} u_{j}^{c}\right),} \\
& {\left[\mathbf{D}_{\mathbf{e}} \mathbf{Y}_{\mathbf{u}}^{\mathbf{T}}\right]^{I P}\left[\mathbf{D}_{\mathbf{e}} \mathbf{Y}_{\mathbf{u}}^{\dagger}\right]^{J S} \frac{1}{m_{S}^{2}}\left(\bar{q}^{c}{ }_{P} i \tau_{2} \ell_{I}\right)\left(\bar{\ell}_{J} i \tau_{2} q_{S}^{c}\right)} & S_{0} \\
& {\left[\mathbf{D}_{\mathbf{u}} \mathbf{K D}_{\mathbf{d}}\right]^{i p}\left[\mathbf{D}_{\mathbf{u}} \mathbf{K}^{*} \mathbf{D}_{\mathbf{d}}\right]^{j s} \frac{1}{m_{S}^{2}}\left(\overline{d^{c}} e_{p}\right)\left(\bar{e}_{j} d_{s}^{c}\right)} & \tilde{S}_{0} \\
\frac{\left|\lambda_{S_{2}}\right|^{2}}{m_{S}^{2}}\left(\bar{u}_{I} \ell_{I}\right)\left(\bar{\ell}_{J} u_{J}\right), & {\left[\mathbf{D}_{\mathbf{e}} \mathbf{Y}_{\mathbf{u}}^{\mathbf{T}}\right]^{j S} \frac{\left|\lambda_{S_{2}}\right|}{m_{S}^{2}}\left(\bar{u}_{I} \ell_{I}\right)\left(\bar{e}_{j} q_{S}\right),} & \\
& {\left[\mathbf{D}_{\mathbf{e}} \mathbf{Y}_{\mathbf{u}}^{\dagger}\right]^{i P}\left[\mathbf{D}_{\mathbf{e}} \mathbf{Y}_{\mathbf{u}}^{\mathbf{T}}\right]^{j S} \frac{1}{m_{S}^{2}}\left(\bar{q}_{P} e_{i}\right)\left(\bar{e}_{j} q_{S}\right)} & S_{2} \\
& {\left[\mathbf{D}_{\mathbf{u}} \mathbf{K}^{*} \mathbf{D}_{\mathbf{d}}\right]^{I p}\left[\mathbf{D}_{\mathbf{u}} \mathbf{K D}_{\mathbf{d}}\right]^{J s} \frac{1}{m_{S}{ }^{2}}\left(\bar{d}_{p} \ell_{I}\right)\left(\bar{\ell}_{J} d_{s}\right)} & \tilde{S}_{2}
\end{array}
$$

where the $\mathbf{Y}_{\mathbf{u}}^{\mathbf{T}}=\mathbf{D}_{\mathbf{u}}\left[=\mathbf{D}_{\mathbf{u}} \mathbf{K}^{*}\right]$ for an up-type [down-type] quark on the external leg. Notice that generation number can only change when down-type quarks are involved.

The amplitudes induced by these operators are suppressed by zero, two or four Yukawa eigenvalues. By construction, the couplings $\propto\left|\lambda_{S_{0}}\right|^{2},\left|\lambda_{S_{2}}\right|^{2}$ of the operators unsuppressed by Yukawa couplings, are generation diagonal, and small enough to satisfy the bounds.

Now, consider the pseudoscalar operators (middle operator of eq. (3.5) and eq. (3.7)), which are suppressed by $y_{f}^{2}$. These operators, mediated by $S_{0}$ or $S_{2}$, always contain an up-type quark and a lepton of the same generation, so they can induce $D_{0} \rightarrow \mu^{ \pm} e^{\mp}$, but not $K_{L} \rightarrow \mu^{ \pm} e^{\mp}$ or $N \mu \rightarrow N e$. Only in the charged current case, where the down-type quark 
brings an element of the CKM matrix $K$, is non-conservation of generation number possible. These operators contribute to the leptonic decay of pseudoscalar mesons $M^{+}=\pi^{+}, K^{+}$or $B^{+}$. Recall that in the SM, the $V-A$ amplitude for $M^{+}$decay is suppressed by a factor of the charged lepton mass, which is required to flip the chirality on the external leg. This suppression is absent for a pseudoscalar operator. The leptoquark $S_{0}$ induces $M^{+} \rightarrow \nu_{\tau} \bar{e}$, with $\varepsilon \sim\left|\lambda_{S_{0}}\right| K_{t m} y_{t} y_{\tau} / 6$, where $m=d, s, b$ as the case may be. This expectation is less than the experimental bound for $\pi$ and $K$ decays, which is $\varepsilon \lesssim 2 \times 10^{-5}$ [14]. The decay $B^{+} \rightarrow \nu e^{+}$is not observed, consistently with its tiny expected SM branching ratio. However, we can compare to the SM prediction for the observed decay $B^{+} \rightarrow \tau^{+} \nu$ :

$$
\frac{B R\left(B^{+} \rightarrow e^{+} \nu_{\tau}\right)}{B R\left(B^{+} \rightarrow \tau^{+} \nu\right)} \simeq \frac{m_{B}^{2}}{m_{\tau}^{2}} \frac{\left|\lambda_{S_{0}} K_{t b} y_{t} y_{\tau}\right|^{2}}{\left|K_{u b}\right|^{2}} \sim 870 y_{\tau}^{2} \sim 870 \frac{m_{\tau}^{2}}{v^{2}} \tan ^{2} \beta
$$

For $\lambda_{S_{0}} \simeq 1 / 4$ and $\tan \beta \gtrsim 1 / \sqrt{2}$, this exceeds the upper bound $B R\left(B^{+} \rightarrow e^{+} \nu\right) \lesssim 4 \times 10^{-2}$ $B R\left(B^{+} \rightarrow \tau^{+} \nu\right)$ by a factor $\sim 2$. So for $\tan \beta=1, B^{+} \rightarrow e^{+} \nu$ provides the best bound on $S_{0}$ with both chiral couplings, in this model. Or, if we allow $\tan \beta$ as a free parameter, $B^{+} \rightarrow e^{+} \nu$ is a sensitive probe for this pattern of couplings for the singlet $S_{0}$.

Charged current pseudoscalar operators are also induced by the doublet leptoquark $S_{2}$, but with different index contractions, such that it induces the observed $B^{+} \rightarrow \nu_{e} \bar{\tau}$ with $\varepsilon \sim\left|\lambda_{S_{2}}\right| K_{t b} y_{t} y_{\tau} / 6$. This contribution can be competitive with the SM, for $\tan \beta \gtrsim 4$, which can be interesting in view of the experimental anomaly in $B R\left(B^{ \pm} \rightarrow \tau^{ \pm} \nu\right)$ (see e.g. [45-47]).

It is easy to check that the operators suppressed by the fouth power of Yukawa eigenvalues are of $V \pm A$ form, and harmless. For instance, experimentally, $K_{L} \rightarrow \bar{\mu} e$ gives one of the most stringent limits on generation-diagonal flavour violation: $\varepsilon \lesssim 3 \times 10^{-7}$. The expectation from $\tilde{S}_{2}$ or $\tilde{S}_{0}$ exchange would be $\varepsilon \sim y_{u} K_{u d} y_{d} y_{c} K_{c s} y_{s} / 6 \sim 2 \tan ^{2} \beta \times 10^{-16}$. Generation number changing operators proportional to $y_{f}^{4}$, neccessarily involve down-type quarks, and are CKM suppressed. For instance for $\mu N \rightarrow e N^{\prime}, \varepsilon \sim\left|y_{u} y_{d} y_{c} K_{c d} y_{d}\right| / 6$ at tree level, which is unobservably small even for large $\tan \beta$. The expectation for $B_{s} \rightarrow \mu \bar{\mu}$ (mediated by $\tilde{S}_{0}$ or $\tilde{S}_{2}$ ) would be $\varepsilon \sim\left|y_{c} K_{c b} y_{b} y_{c} K_{c s} y_{s}\right| / 6$, which is much less than the experimental limit [14] of $7 \times 10^{-5}$. Since lepton generation change occurs via CKM mixing angles, which appear in $\lambda$ suppressed by quark Yukawa eigenvalues, $\tau \rightarrow \mu \gamma$ is more sensitive than $\mu \rightarrow e \gamma$ because it involves higher generations. The $\tau \rightarrow \mu \gamma$ loop has an internal down-type quark, and gives [38] (for $\tilde{S}_{0}$ - the factor of $1 / 6$ is a function of the charges of the loop particles):

$$
\varepsilon \sim \frac{1}{6} \frac{N_{c}}{16 \pi^{2}} y_{t} y_{c} \sum_{r} K_{t r} y_{d, r}^{2} K_{c r} \sim \frac{1}{96 \pi} y_{c} y_{b}^{2} K_{c b}
$$

which is less than the experimental bound (see table 2). In the last approximation of eq. (3.10), and in all the tables, we approximate $\pi \simeq N_{c}$, and $y_{t} \simeq K_{i i} \simeq 1$. The most sensitive process we found was $K^{+} \rightarrow \pi^{+} \nu_{\tau} \bar{\nu}_{\tau}$ due to $S_{0}$ exchange, for which we estimate

$$
6 \varepsilon \sim y_{\tau}^{2} y_{t}^{2} K_{t d} K_{t s}, \quad\left(y_{t}^{2} K_{t d} K_{t s} y_{d} y_{s}\right)<5 \times\left. 10^{-5}\right|_{\operatorname{expt}}
$$

which is in agreement with the bounds for $\tan \beta \lesssim 45$ (in parentheses is the estimate for $\tilde{S}_{2}$ exchange). 
Table 2 lists some interesting rare processes, with the experimental bounds, and, in the third colomn, the largest rates mediated by any of the leptoquarks with this pattern of couplings. These estimates suggest that our "unflavoured" leptoquarks $S_{0}$ or $S_{2}$ could be found in leptonic $B$ decays $B^{+} \rightarrow l^{+} \nu$, and that the rare process most sensitive to $\tilde{S}_{0}$ is $\tau \rightarrow \mu \gamma$.

Alternatively, hadron colliders could search for all these leptoquarks, which would decay near the production point as assumed in Tevatron searches. $S_{0}$ and $S_{2}$ decay with similar branching ratios to $e u, \mu c$ and $\tau t$, and $\tilde{S}_{0}$ and $\tilde{S}_{2}$ would preferentially decay to third generation fermions.

\subsection{Some comments}

In our estimates, and in the table 2 , we quote the largest rate mediated by any of the leptoquarks. This is not because all the leptoquarks are present with $m_{S} \sim 300 \mathrm{GeV}$, but rather that we prefer to present one concise table, rather than one for each of the six leptoquark couplings. In this section, we assume the presence at the "flavour scale" of the new flavour structures ("spurions") corresponding to the identity matrix linking the $u$-type and lepton flavour spaces. With these new spurions, we can construct $\lambda$ matrices for any leptoquark and guestimate the induced rates. The largest rates, for any leptoquark, are in the table. So if only one, or some of the leptoquarks are light, not all our guestimates will be fulfilled. For example, the bound on $\tan \beta$ from eq. (3.9) only applies if there is an $S_{0}$ with both chiral couplings and $m_{S} \sim 300 \mathrm{GeV}$.

Rather than assuming that the new spurion was the identity matrix, with a coefficient $\lambda_{S_{0,2}}$ that we are free to ajust, we could take the approach that the spurions were $\lambda_{S_{0}} \mathcal{I}$ and $\lambda_{S_{2}} \mathcal{I}$. Then the operator coefficients in eq. (3.5) to eq. (3.8) would all be proportional to the same coefficient $\lambda_{S_{0,2}}^{2} / m_{S}^{2}$. In table 2 , this would multiply the coefficients of pseudoscalar operators (above the double bar) by $\lambda_{S_{0,2}} \sim 1 / 4$, and the coefficients of vector operators (below the double bar) would be multiplied by $\lambda_{S_{0,2}}^{2} \sim 1 / 16$. The ratio eq. (3.9) is suppressed by a further factor of order of $\lambda_{S_{0}}^{2} \leq 1 / 16$, making the upper bound on $\operatorname{Br}\left(B^{+} \rightarrow e^{+} \nu\right)$ compatible with $\tan \beta=O(1)$. The contributions from the operators suppressed by four powers of Yukawa couplings, already negligible, are even more suppressed in this case. The most sensitive probe would be $K^{+} \rightarrow \pi^{+} \nu_{\tau} \bar{\nu}_{\tau}$, in agreement with the current bound for all values of $\tan \beta$.

An interesting question which models can address, is the relative sensitivity of $B$ and $K$ decays to leptoquarks. The experimental bounds on the various $\varepsilon$ s arising from $K$ decays are lower than those from $B$ mesons. However, leptoquarks could have larger couplings to third generation fermions, resulting in larger contributions in $B$ decays than $K$ decays. As discussed around eq. (3.9), the $\mathcal{O}\left(y_{f}^{2}\right)$ charged current pseudoscalar decays, which can be $\propto y_{\tau} K_{t x}$, are more tightly constrained by $B$ decays than by $K$ mesons, whereas the best bound on the $\mathcal{O}\left(y_{f}^{4}\right)$ operators, which can be $\propto y_{\tau}^{2} K_{t x} K_{t y}$, is from $K \rightarrow \pi \nu \bar{\nu}$. This illustrates the interest of this decay for Beyond the Standard Model physics: it can probe the interactions of third generation leptons, and also of the top via the CKM matrix.

The final issue is the relative importance of loop and tree diagrams. With the hierarchical couplings we consider, it is possible that (third generation) loops dominate over (first generation) tree level processes. For instance, this occurs in $\mu N \rightarrow e N^{\prime}$, which is well 


\begin{tabular}{|c|c|c|}
\hline process & $\varepsilon<$ & estimates (unflavoured S) \\
\hline$R_{K}$ & $2 \times 10^{-5}$ & $\frac{1}{6}\left|\lambda_{S_{0}}\right| K_{t s} y_{t} y_{\tau} \sim 2 \times 10^{-5} t_{\beta}$ \\
\hline$B^{+} \rightarrow e^{+} \nu$ & $2 \times 10^{-4}$ & $\frac{1}{6}\left|\lambda_{S_{0}}\right| y_{\tau} y_{t} K_{t b} \sim 3 \times 10^{-4} t_{\beta}$ \\
\hline$B^{+} \rightarrow \tau^{+} \nu$ & $8 \times 10^{-4}$ & $\frac{1}{6}\left|\lambda_{S_{2}}\right| y_{\tau} y_{t} K_{t b} \sim 3 \times 10^{-4} t_{\beta}$ \\
\hline$D_{0} \rightarrow \mu^{ \pm} e^{\mp}$ & $6 \times 10^{-4}$ & $\frac{1}{6}\left|\lambda_{S_{0}}\right| y_{\mu} y_{c} \sim 2 \times 10^{-7} t_{\beta}$ \\
\hline$K^{+} \rightarrow \pi^{+} \nu \bar{\nu}$ & $9 \times 10^{-6}$ & $\frac{1}{6} y_{\tau}^{2} y_{t}^{2} K_{t s} K_{t d} \sim 5 \times 10^{-9} t_{\beta}^{2}$ \\
\hline$K_{L} \rightarrow \mu^{ \pm} e^{\mp}$ & $3 \times 10^{-7}$ & $\frac{1}{6} y_{u} y_{d} y_{c} y_{s} \sim 2 \times 10^{-16} t_{\beta}^{2}$ \\
\hline$B_{s} \rightarrow \mu^{ \pm} \mu^{\mp}$ & $7 \times 10^{-5}$ & $\frac{1}{6} y_{c}^{2} K_{c b} y_{b} y_{s} \sim 5 \times 10^{-12} t_{\beta}^{2}$ \\
\hline$B^{+} \rightarrow K^{+} \tau^{ \pm} \mu^{\mp}$ & $2 \times 10^{-3}$ & $\frac{1}{6} y_{t} y_{b} y_{c} y_{s} \sim 2 \times 10^{-8} t_{\beta}^{2}$ \\
\hline$\mu N \rightarrow e N^{\prime}$ & $8 \times 10^{-7}$ & $\frac{1}{96 \pi^{2}} y_{c} y_{u} K_{c b} y_{b}^{2} K_{u b} \sim 10^{-17} t_{\beta}^{2}$ \\
\hline$\mu \rightarrow e \gamma$ & $\sim 10^{-6}$ & $\frac{1}{96 \pi} y_{c} y_{u} K_{c b} y_{b}^{2} K_{u b} \sim 2 \times 10^{-17} t_{\beta}^{2}$ \\
\hline$\tau \rightarrow \mu \gamma$ & $10^{-4}$ & $\frac{1}{96 \pi} y_{c} y_{t} K_{c b} y_{b}^{2} K_{t b} \sim 7 \times 10^{-10} t_{\beta}^{2}$ \\
\hline
\end{tabular}

Table 2. The largest predicted coefficient $\varepsilon$, induced by any of the scalar leptoquarks we consider, of mass $\sim 300 \mathrm{GeV}$, with the flavoured coupling $\lambda^{\mathrm{LQ}}$ following the patterns considered in section 3 . The second colomn is the bound on $\varepsilon$ (defined in eq. (2.7)) for the process in the first colomn. The bounds above the double line are on pseudoscalar operators (which can be induced by the two interactions of $S_{0}$ and $S_{2}$ ), those below are $V \pm A$. In the third colomn, $\lambda_{S_{0}}, \lambda_{S_{2}} \lesssim 0.25$. The expectation quoted for $\mu-e$ conversion is from a loop diagram; the tree level expectation is smaller, as discussed around eq. (3.13). 

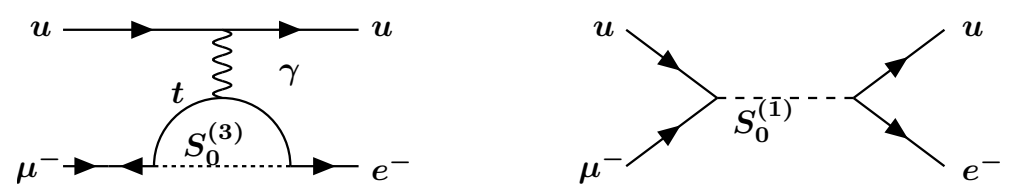

Figure 2. Loop and tree leptoquark contributions to $\mu N \rightarrow e N^{\prime}$. The diagram represents leptoquarks with quark flavour (= generation number), as considered in section 4 . For the various patterns of $\lambda$ 's that we consider, the loop diagram involving third generation quarks dominates.

known to be a sensitive probe of the effective $\mu-e-\gamma$ vertex. The tree level amplitudes for $\mu N \rightarrow e N^{\prime}$ give

$$
6 \epsilon \simeq\left\{\begin{array}{lrl}
y_{u} y_{d} y_{c} K_{c d} y_{d} & \sim 2 \times 10^{-18} t_{\beta}^{2} & \left(\tilde{S}_{2}, \tilde{S}_{0}\right) \\
y_{e} y_{u} y_{\mu} y_{c} K_{c d} & \sim 10^{-18} t_{\beta}^{2} & \left(S_{2}\right)
\end{array}\right.
$$

and are smaller than loop leptoquark exchange (see the diagrams of figure 2). We take the loop contribution to be $[12]^{2}\left|\varepsilon^{e \mu}\right|^{2} \times \alpha_{\mathrm{em}} \log \left(m_{W}^{2} / m_{\mu}^{2}\right)$, and obtain the loop to tree ratio (mediated by $\tilde{S}_{0}$ ):

$$
\alpha_{\mathrm{em}} \log \left(\frac{m_{W}}{m_{\mu}}\right)^{2}\left|\frac{\frac{N_{c}}{96 \pi^{2}} y_{c} y_{u} K_{c b} y_{b}^{2} K_{u b}}{y_{u} y_{d} y_{c} y_{d} K_{c d}}\right|^{2} \sim 10^{-3}\left|\frac{K_{c b} y_{b}^{2} K_{u b}}{y_{d}^{2} K_{c d}}\right|^{2} \gtrsim 10 .
$$

In table 2, we quote the loop expectation.

It can also arise in the SM that third generation loops exceed tree level amplitudes (for instance in $\bar{p} p \rightarrow$ Higgs). This problem is exacerbated for our $\lambda$ couplings, because they can be proportional to (Yukawa coupling) ${ }^{n}$, for $n \geq 2$. This raises two questions. First, have we considered the most restrictive loop-induced processes, and secondly, are there loop contributions which exceed the tree estimates in the tables. With respect to the first question, we expect that the most sensitive one loop diagrams will have external lepton legs and an internal leptoquark and $t$ or $b$ loop, because the coloured loop is enhanced by $N_{c}$, and there are several strict limits on New Physics in the lepton sector. So $\tau \rightarrow \mu \gamma$, $\mu \rightarrow e \gamma$ and $g-2$ should give the best limits. We did not consider the box contributions to meson-anti-meson mixing, which would be proportional to Yukawa eigenvalues for the external quarks and for the internal (third generation) leptons.

It is difficult to ascertain whether there could be loop diagrams larger than our tree estimates. To avoid the suppression present in the tree amplitudes, due to small Yukawa eigenvalues, the leptoquark should not interact with the external leg fermions. This would not be possible for the lepton flavour violating decays, because the lepton flavour violation is provided by the leptoquarks. In table 2 , that leaves $K^{+} \rightarrow \pi^{+} \nu \bar{\nu}$ and $B_{s} \rightarrow \mu^{+} \mu^{-}$. An SM loop would be required to induce the flavour off-diagonal quark current, so the leptoquark loop would merely modify flavour diagonal lepton interactions already present in the SM. Such leptoquark loops should be better constrained by precision observables, such as $g-2$, which probe lepton interactions more directly. So even if there is a loop contribution that exceeds our tree estimates, we doubt that it would be phenomenologically relevant. 


\section{Leptoquarks with quark flavour}

In R-parity violating supersymmetric (RPV SUSY) theories, the squarks can have interactions with quarks and leptons. Insofar as a leptoquark is a boson interacting with a lepton and a quark, such squarks can therefore be identified as leptoquarks (with a quark flavour index). An implementation of the MFV hypothesis in RPV SUSY seesaw models has recently been investigated by Nikolidakis and Smith [23], who showed that the lepton number violating couplings were sufficiently suppressed that $R$-parity was not required to ensure proton stability.

The idea used by Nikolidakis and Smith to obtain spurions with a single lepton flavour index, was the cross product. Since SM flavour spaces are 3 dimensional, the fully antisymmetric $\varepsilon_{I J K}$ tensor can be contracted with an anti-symmetric two-index object (such as $Y_{e} Y_{e}^{\dagger}\left[m_{\nu}\right]$, where $\left[m_{\nu}\right]$ is the symmetric majorana mass matrix of the light neutrinos) to obtain a spurion with a singlet lepton index

$$
\bar{\Upsilon}_{I} \equiv \frac{1}{m_{a t m}} \varepsilon_{I J K}\left[Y_{e} Y_{e}^{\dagger} m_{\nu}\right]^{J K}
$$

where $m_{\mathrm{atm}}=\sqrt{\Delta m_{\mathrm{atm}}^{2}} \simeq .05 \mathrm{eV}$ is the atmospheric mass difference which we take as the neutrino mass scale.

This formula requires some discussion, because it is phenomenologically peculiar to promote the coefficient of the non-renormalisable operator $\left[m_{\nu}\right]$ to the status of fundamental flavour structure (or spurion): non-renormalisable operators are not spurions in the quark sector, where MFV is approximately confirmed by the data. It is also theoretically peculiar: one can anticipate that the flavour pattern was generated at some high scale, and transmited to low energy via renormalisable couplings. So the flavour pattern in $\left[m_{\nu}\right]$ can arise from the product of several spurions (as in the seesaw mechanism), and the overall magnitude is controlled by a ratio of energy scales, which may have nothing to do with the flavour structure.

We will use $\left[m_{\nu}\right]$ as a spurion anyway, because a product of spurions is also a spurion, the neutrino mass matrix is the only available information about lepton flavour violation, and an object with two indices in lepton doublet space is required to contract with $\varepsilon_{I J K}$. However, we normalise to the light neutrino mass scale $m_{\mathrm{atm}}$, rather than to the Higgs vacuum expectation value as in [23], because we think MFV is about the flavour pattern, whereas majorana neutrino masses may be small because they violate lepton number. ${ }^{7}$ Phrased another way: the ratio $\langle H\rangle / M_{\nu_{R}}$ in the seesaw mechanism may have nothing to do with flavour.

For hierarchical ${ }^{8}\left[m_{\nu}\right]_{I J}$, and tri-bi-maximal mixing $[44],{ }^{6}$ this gives

$$
\bar{\Upsilon}^{I}=\frac{\varepsilon^{I J K}\left[Y_{e} Y_{e}^{\dagger} m_{\nu}\right]_{J K}}{m_{\mathrm{atm}}}=\frac{1}{8}\left[\begin{array}{c}
\left(y_{\mu}^{2}-y_{\tau}^{2}\right)(4-3 \delta) \\
-\sqrt{2}\left(y_{\tau}^{2}-y_{e}^{2}\right)\left(\sqrt{3} \delta-4 s_{13}\right) \\
-\sqrt{2}\left(y_{\mu}^{2}-y_{e}^{2}\right)\left(\sqrt{3} \delta+4 s_{13}\right)
\end{array}\right] \sim-\frac{1}{2}\left[\begin{array}{c}
y_{\tau}^{2} \\
y_{\tau}^{2} \delta \\
y_{\mu}^{2} \delta
\end{array}\right]
$$

\footnotetext{
${ }^{7}$ With our normalisation, the demonstration of [23] that MFV suppresses RPV sufficiently would no longer hold.

${ }^{8}$ For the inverse hierarchy, we would obtain $\bar{\Upsilon} \sim y_{\tau}^{2}\left(1, \delta^{2}, \delta^{2} y_{\mu}^{2} / y_{\tau}^{2}\right)$.
} 
for $\delta \equiv m_{\text {sol }} / m_{\text {atm }} \simeq 1 / 6$ and $s_{13}=\sin \theta_{13} \lesssim .1$. We can already anticipate that in this pattern, a leptoquark interacts with all flavours of leptons, and most strongly to the first and second generation doublets (there will be an additional $Y_{e}$ in the couplings to singlet charged leptons). This is unsurprising, since the lepton flavour violation is related to the large mixing angles of the lepton mixing matrix. However, lepton flavour changing rates are suppressed by at least a factor $y_{\tau}^{4}$.

The spurion $\bar{\Upsilon}_{I}$, of the authors of [23], which has only one lepton doublet index, allowed them to construct the $R$-parity violating $\lambda^{\prime} L Q D^{c}$ as

$$
\lambda^{\prime} \propto \bar{\Upsilon} Y_{d}
$$

In an analogous fashion, using $\bar{\Upsilon}_{I}$, we can construct the $\lambda$ couplings of leptoquarks carrying quark generation number. To do this, we must assign the leptoquark to live in one of the quark flavour spaces. A possibility would be for the leptoquark to carry the flavour of the quark with which it interacts, in which case no quark Yukawa matrix is required at the vertex (the identity matrix is sufficient). Since we explicitly wish the leptoquark couplings to have flavour structure, we do not consider this option - although it is interesting because it could give large couplings. We discuss two possibilities below, where the leptoquark coupling matrices are proportional to quark Yukawa matrices.

\subsection{Maximal coupling}

The largest leptoquark couplings are obtained by allowing only one power of the quark Yukawa coupling at the vertex, selected according to the type of quark. Suppose, for instance, that the leptoquark interacts with singlet $d$ quarks. Then $Y_{d}$ imposes that $S$ has doublet quark flavour indices. With this "maximum coupling" hypothesis, we obtain

$$
\begin{aligned}
& \lambda_{\mathbf{L} \mathbf{S}_{\mathbf{0}}} S_{0} \bar{\ell} i \tau_{2} q^{c} \rightarrow\left[\overline{\mathbf{\Upsilon}}^{*}\right]^{I}\left[\mathbf{Y}_{\mathbf{d}, \mathbf{u}}^{\dagger}\right]^{r P} S_{0}^{r} \bar{\ell}_{I} i \tau_{2} q_{P}^{c} \quad S_{0} \sim d, u \\
& \lambda_{\mathbf{R S}_{\mathbf{0}}} S_{0} \bar{e} u^{c} \rightarrow\left[\mathbf{Y}_{\mathbf{e}}^{\mathbf{T}} \overline{\mathbf{\Upsilon}}^{*}\right]^{i}\left[\mathbf{Y}_{\mathbf{u}}\right]^{P q} S_{0}^{P} \bar{e}_{i} u_{q}^{c} \quad S_{0} \sim q \\
& \tilde{\lambda}_{\mathbf{R} \tilde{\mathbf{S}}_{\mathbf{0}}} \tilde{S}_{0} \bar{e} d^{c} \rightarrow\left[\mathbf{Y}_{\mathbf{e}}^{\mathbf{T}} \overline{\mathbf{\Upsilon}}^{*}\right]^{\mathbf{i}}\left[\mathbf{Y}_{\mathbf{d}}\right]^{\operatorname{Pr}} \tilde{S}_{0}^{P} \bar{e}_{i} d_{r}^{c} \quad \tilde{S}_{0} \sim q \\
& \lambda_{\mathbf{L S}} S_{2} \bar{\ell} u \rightarrow\left[\overline{\mathbf{\Upsilon}}^{*}\right]^{I}\left[\mathbf{Y}_{\mathbf{u}}^{*}\right]^{P r} S_{2}^{P \bar{\ell}_{I}} u_{r} \quad S_{2} \sim \bar{q} \\
& \lambda_{\mathbf{R S}_{2}} S_{2} \bar{e}\left[i \tau_{2} q\right] \rightarrow\left[\mathbf{Y}_{\mathbf{e}}^{\mathbf{T}} \overline{\mathbf{\Upsilon}}^{*}\right]^{i}\left[\mathbf{Y}_{\mathbf{u}, \mathbf{d}}^{\mathbf{T}}\right]^{r P} S_{2}^{r} \bar{e}_{i}\left[i \tau_{2} q_{P}\right] \quad S_{2} \sim \bar{u} \text { or } \bar{d} \\
& \tilde{\lambda}_{\mathbf{L} \tilde{\mathbf{S}}_{\mathbf{2}}} \tilde{S}_{2} \bar{\ell} d \rightarrow\left[\overline{\mathbf{\Upsilon}}^{*}\right]^{I}\left[\mathbf{Y}_{\mathbf{d}}^{*}\right]^{P r} \tilde{S}_{2}^{P \bar{\ell}_{I}} d_{r} \quad \tilde{S}_{2} \sim \bar{q}
\end{aligned}
$$

where we added the appropriate quark flavour indices to the leptoquarks, and indicate in the last colomn the flavour space they live in. The interactions involving the doublet quarks $\lambda_{\mathbf{L S}_{\mathbf{0}}}, \lambda_{\mathbf{R S}_{\mathbf{2}}}$ can be taken $\propto \mathbf{Y}_{\mathbf{d}}$ or $\mathbf{Y}_{\mathbf{u}}$; we take $\mathbf{Y}_{\mathbf{u}}$ because the eigenvalues are larger. The leptoquarks $S_{0}$ and $S_{2}$ both can have two distinct interactions. In this "maximal" pattern, the two interactions assign the leptoquarks to different flavour spaces, which disfavours this pattern, or the presence of both couplings.

This naive attempt to obtain large couplings leads to a peculiar behaviour: the $U_{Y}(1)$ charges of the leptoquarks do not match those of the corresponding quarks with the same flavour structure. This is not an internal inconsistency: the original $\mathrm{U}(3)^{5}$ symmetry can 
be decomposed into five distinct $\mathrm{U}(1)$ and $\mathrm{SU}(3)$ subgroups [26], and we are not forced to choose the same $\mathrm{SU}(3)$ and $\mathrm{U}(1)$ assignments for quarks and leptoquarks. However, it is clearly an unusual choice. A flavour assignment for the leptoquarks that match their gauge quantum numbers will be discussed in the next subsection.

In this pattern, all generations of leptoquarks have similiar couplings $\left(\lesssim y_{\tau}^{2} y_{t}\right)$ to all flavours of leptons. However, the couplings to quarks are hierarchical and generation diagonal (between the quark and leptoquark), up to insertions of CKM matrix elements. For instance, third generation leptoquarks only interact with third generation quarks, and doublet down-type quarks of the first and second generations via CKM-suppressed terms.

The absence of a spurion which links quarks and leptons has two consequences: fermion generation diagonal interactions are not favoured, and the Yukawa suppression of quark and lepton bilinears can be studied separately. The $\varepsilon$ factor (as defined in eq. (2.7)) for a dimension six operator formed from the product of two bilinears, will be the product of the coefficients given for the two bilinears. The quark bilinears induced by leptoquarks of generation $T$ (or $t$ ), with their coefficients, are:

$$
\begin{array}{llr}
K_{T P}^{*} y_{u, T}^{2} K_{T S}\left(\bar{d}_{P} \gamma^{\mu} P_{L} d_{S}\right), K_{T S}^{*} y_{u, T}^{2}\left(\bar{d}_{S} \gamma^{\mu} P_{L} u_{T}\right), y_{u, T}^{2}\left(\bar{u}_{T} \gamma^{\mu} P_{L} u_{T}\right), y_{u, t}^{2}\left(\bar{u}_{t} \gamma^{\mu} P_{R} u_{t}\right) & S_{0} \\
K_{T P}^{*} y_{u, T}^{2} K_{T S}\left(\bar{d}_{P} \gamma^{\mu} P_{L} d_{S}\right), y_{u, T}^{2}\left(\bar{u}_{T} \gamma^{\mu} P_{L} u_{T}\right), & y_{u, t}^{2}\left(\bar{u}_{t} \gamma^{\mu} P_{R} u_{t}\right), & S_{2} \\
y_{d, t}^{2}\left(\bar{d}_{t} \gamma^{\mu} P_{R} d_{t}\right), & & \tilde{S}_{2}, \tilde{S}_{0}
\end{array}
$$

where $s, t(T, P, S)$ are singlet (doublet) generation labels, not to be summed over. As expected, since we construct the quark flavour structure of the leptoquark coupling with SM Yukawa matrices, we find an MFV-like suppression: quark flavour change can only occur in charged current interactions, or among the $d_{L} \mathrm{~S}$. Since the two possible interactions of $S_{0}$ and $S_{2}$ assign the leptoquark to different flavour spaces, they cannot be simultaneous present, and bilinears like $\bar{q} d$, or $\bar{\ell} \sigma e F$ cannot be generated (except with an external mass insertion).

The lepton bilinears, with the flavour factors of their coefficients, are

$$
\bar{\Upsilon}^{I *} \bar{\Upsilon}^{J}\left(\bar{\ell}_{I} \gamma^{\mu} \ell_{J}\right), \quad y_{e, i} y_{e, j} \bar{\Upsilon}^{i *} \bar{\Upsilon}^{j}\left(\bar{e}_{i} \gamma^{\mu} e_{j}\right)
$$

where $I, J, i, j$ are not summed. Lepton flavour violation is suppressed by an extra lepton Yukawa coupling in the singlets, so focussing on the doublet bilinears which are all suppressed by an overall $y_{\tau}^{4} / 4$, the relative ratio of flavours is ${ }^{9}$

$$
e e: e \mu: e \tau: \mu \mu: \mu \tau=1: \delta:\left(\frac{m_{\mu}}{m_{\tau}}\right)^{2} \delta: \delta^{2}: \delta^{2}\left(\frac{m_{\mu}}{m_{\tau}}\right)^{2}
$$

where $\delta \simeq 1 / 6$ is defined after eq. (4.2). This pattern predicts $B R(\mu \rightarrow e \gamma)>B R(\tau \rightarrow$ $\mu \gamma) / B R(\tau \rightarrow \mu \nu \bar{\nu})$, and for a top and third generation $\left(S_{2}\right)$ leptoquark in the loop,

$$
\varepsilon_{L, R}^{e \mu} \sim \frac{1}{4} \frac{N_{c}}{64 \pi^{2}} y_{\tau}^{4} y_{t}^{2} \delta \simeq 2 \times 10^{-4} \frac{m_{\tau}^{4}}{v^{4}} \tan ^{4} \beta
$$

\footnotetext{
${ }^{9}$ For the inverse hierarchy, we would obtain $e e: e \mu: e \tau: \mu \mu: \mu \tau \sim 1: \delta^{2}: \delta^{2}\left(\frac{m_{\mu}}{m_{\tau}}\right)^{2}: \delta^{4}: \delta^{4}\left(\frac{m_{\mu}}{m_{\tau}}\right)^{2}$, which is sufficiently similar that we do not consider it further.
} 
For $S_{0}$ in the loop, we estimate [38] $\varepsilon_{L, R}^{e \mu}$ a factor $\sim 1 / 3$ smaller. This is within the current experimental bound $\varepsilon \lesssim 10^{-6}$ for $\tan \beta \lesssim 25$. This dipole induced by the third generation leptoquark, will give the dominant contribution to $\mu N \rightarrow e N^{\prime}$ (see eq. 3.13) because the tree level exchange of a first generation leptoquark is suppressed by first generation quark Yukawa couplings (we estimate the tree-level $\varepsilon \sim y_{\tau}^{4} \delta y_{d}^{2} / 24$ ).

In table 3, various rare decays are estimated. Generation number is not conserved in this pattern, so the experimentally prefered rare decay $B_{s} \rightarrow \mu \bar{\mu}$ is also the more sensitive one

$$
\frac{B R\left(B_{s} \rightarrow \mu^{ \pm} \tau^{\mp}\right)}{B R\left(B_{s} \rightarrow \mu^{ \pm} \mu^{\mp}\right)} \sim \frac{\left|y_{\mu} y_{\tau} y_{\tau}^{2} y_{\mu}^{2} \delta^{2} K_{t s} K_{t b}\right|^{2}}{\left|y_{\mu}^{2} y_{\tau}^{4} \delta^{2} K_{t s} K_{t b}\right|^{2}} \ll 1
$$

However, the meson decay rates in this pattern are very small. From eq. (4.10), one sees that no flavour change in induced at tree level among singlet quarks, or up-type doublet components. The FCNC decays of $K$ and $B$ mesons only occur through the first bilinear of eq. (4.10), which combines with $\left(\bar{\nu} \gamma_{\mu} P_{L} \nu\right)$ or $\left(\bar{e} \gamma_{\mu} P_{R} e\right)$ in the four fermion interactions induced at tree level by $S_{0}$ s and $S_{2}$ s (see table 1). Hence in eq. (4.14), the charged leptons are singlets, and have the additional $y_{e, i} y_{e, j}$ factor of the second bilinear of eq. (4.11). As can be seen from table 3, the least suppressed meson decay is $K^{+} \rightarrow \pi^{+} \nu \bar{\nu}$, mediated by $S_{0}$, which reaches the experimental bound for $\tan \beta \sim 100$. However, as shown in eq. (4.13), $\mu \rightarrow e \gamma$, which can also be mediated by $S_{0}$, is detectable for $\tan \beta \sim 35$.

The most promising precision searches for this pattern of leptoquark couplings would be $\mu \rightarrow e \gamma\left(\right.$ or $\left.\mu N \rightarrow e N^{\prime}\right)$.

At hadron colliders, leptoquarks can be produced via strong interactions, and decay via their $\lambda$ couplings. Searches frequently suppose that $\lambda \gtrsim 10^{-8}$, so that the leptoquark decays within a few centimetres of its production point. This condition is verified, for $\tan \beta \geq 1$, for all the third generation leptoquarks in this pattern except $\tilde{S}_{0}$ (which requires $\tan \beta \gtrsim 2$ ). So searches for leptoquarks decaying to a $t$ or $b$ are interesting. The third generation quark would be accompagnied by an electron or a muon, due to the comparatively democratic coupling to leptons in this pattern, as can be seen from equations (4.4) to (4.9).

\subsection{Coherent gauge and flavour assignments}

In this section, we suppose that the flavour space of the leptoquarks is determined by their gauge couplings. For instance, doublet leptoquarks should be in the $q$ flavour space, and the hypercharge of $S_{0}$ implies that it should live in $d$ space. This suggests the following leptoquark interactions:

$$
\begin{aligned}
\lambda_{\mathbf{L S}} S_{0} \bar{\ell}_{0} i \tau_{2} q^{c} & \rightarrow\left[\overline{\mathbf{\Upsilon}}^{*}\right]^{I}\left[\mathbf{Y}_{\mathbf{d}}^{\dagger}\right]^{r P} S_{0}^{r} \bar{\ell}_{I} i \tau_{2} q_{P}^{c} & S_{0} \sim d \\
\lambda_{\mathbf{R S}_{\mathbf{0}}} S_{0} \bar{e} u^{c} & \rightarrow\left[\mathbf{Y}_{\mathbf{e}}^{\mathbf{T}} \overline{\mathbf{\Upsilon}}^{*}\right]^{i}\left[\mathbf{Y}_{\mathbf{d}}^{\dagger} \mathbf{Y}_{\mathbf{u}}\right]^{p r} S_{0}^{p} \bar{e}_{i} u_{r}^{c} & S_{0} \sim d \\
\tilde{\lambda}_{\mathbf{L} \tilde{\mathbf{S}}_{\mathbf{2}}} \tilde{S}_{2} \bar{\ell} d & \rightarrow\left[\overline{\mathbf{\Upsilon}}^{*}\right]^{I}\left[\mathbf{Y}_{\mathbf{d}}^{*}\right]^{P r} \tilde{S}_{2}^{P} \bar{\ell}_{I} d_{r} & \tilde{S}_{2} \sim \bar{q}
\end{aligned}
$$

The hypercharge of $S_{2}$ and $\tilde{S}_{0}$ do not match that of any SM coloured particles, so we do not consider them further. So $S_{0}$ and $\tilde{S}_{2}$ ressemble, respectively, a singlet $d$ squark, and a anti-squark doublet, and the interactions of these "leptoquarks" should correspond to 


\begin{tabular}{|l|l|l|l|}
\hline process & $\varepsilon<$ & S with quark flav (max) & S with quark flav (gauge) \\
\hline$D^{+} \rightarrow \pi^{+} \mu^{ \pm} e^{\mp}$ & $2 \times 10^{-2}$ & & $\frac{1}{24} K_{c b} y_{b}^{2} K_{u b} y_{\tau}^{4} \delta \sim 7 \times 10^{-18} t_{\beta}^{6}$ \\
\hline$K^{+} \rightarrow \pi^{+} \nu \bar{\nu}$ & $9 \times 10^{-6}$ & $\frac{1}{24} y_{\tau}^{4} y_{t}^{2} K_{t s} K_{t d} \sim 10^{-13} t_{\beta}^{4}$ & \\
\hline$K_{L} \rightarrow \mu^{ \pm} e^{\mp}$ & $3 \times 10^{-7}$ & $\frac{1}{24} y_{\tau}^{4} y_{\mu} y_{e} \delta K_{t s} y_{t}^{2} K_{t d} \sim 5 \times 10^{-23} t_{\beta}^{6}$ & \\
\hline$B_{s} \rightarrow \mu^{ \pm} \mu^{\mp}$ & $7 \times 10^{-5}$ & $\frac{1}{24} y_{\tau}^{4} y_{\mu}^{2} \delta^{2} K_{t s} y_{t}^{2} K_{t b} \sim 10^{-19} t_{\beta}^{6}$ & \\
\hline$B^{+} \rightarrow K^{+} \tau^{ \pm} \mu^{\mp}$ & $2 \times 10^{-3}$ & $\frac{1}{24} y_{\tau}^{3} y_{\mu}^{3} \delta^{2} K_{t s} y_{t}^{2} K_{t b} \sim 10^{-20} t_{\beta}^{6}$ & \\
\hline$\mu N \rightarrow e N^{\prime}$ & $8 \times 10^{-7}$ & $\frac{1}{256 \pi^{2}} y_{\tau}^{4} y_{t}^{2} \delta \simeq 10^{-12} t_{\beta}^{4}$ & $\frac{1}{16 \pi^{2}} t_{\beta} y_{\tau}^{4} \delta y_{b}^{2} \sim 8 \times 10^{-15} t_{\beta}^{7}$ \\
\hline$\mu \rightarrow e \gamma$ & $\sim 10^{-6}$ & $\frac{1}{256 \pi} y_{\tau}^{4} \delta \sim 2 \times 10^{-12} t_{\beta}^{4}$ & $\frac{1}{16 \pi} t_{\beta} y_{\tau}^{4} \delta y_{b}^{2} \sim 2 \times 10^{-14} t_{\beta}^{7}$ \\
\hline$\tau \rightarrow \mu \gamma$ & $\sim 10^{-4}$ & $\frac{1}{256 \pi} y_{\tau}^{2} y_{\mu}^{2} \delta^{2} \sim 10^{-15} t_{\beta}^{4}$ & $\frac{1}{16 \pi} t_{\beta} y_{\tau}^{2} y_{\mu}^{2} \delta^{2} y_{b}^{2} \sim 8 \times 10^{-18} t_{\beta}^{7}$ \\
\hline
\end{tabular}

Table 3. Predicted coefficients $\varepsilon$, induced by a scalar leptoquark of mass $\sim 300 \mathrm{GeV}$, with the flavoured coupling $\lambda^{\mathrm{LQ}}$ arising when leptoquarks carry quark flavour. The second colomn is the bound on $\varepsilon$ (defined in eq. (2.7)) for the process in the first colomn, and the third and fourth colomns are the largest expected values of $\varepsilon$ mediated by any of the leptoquarks, for respectively the cases considered in sections 4.1 and 4.2 .

those of squarks with the R-parity violating superpotential $W \supset \lambda_{L}^{\prime} L Q D^{c}+\lambda_{R}^{\prime} D^{c *} E^{c} U^{c}$ (where we allow the non-holomorphic interaction corresponding to $\lambda_{R S_{0}}$ ).

The coefficients of the lepton bilinears of eq. (4.11) remain the same. The chirality flipping dipole operator $\bar{\ell}_{J} \sigma^{\alpha \beta} e_{i} F_{\alpha \beta}$ can now arise, due to the simultaneous presence of $\lambda_{L S_{0}}$, and $\lambda_{R S_{0}}$. From eq. (2.11), with a top and third generation leptoquark in the loop:

$$
\varepsilon \sim 4 \frac{m_{t}}{m_{e, i}} \frac{N_{c}}{16 \pi^{2}} \bar{\Upsilon}^{J} \bar{\Upsilon}^{i} y_{e, i} y_{b}^{2} \rightarrow \tan \beta\left[y_{b}^{2} \frac{N_{c}}{16 \pi^{2}} y_{\tau}^{4} \delta\right]
$$

where after the arrow is the expectation for $\mu \rightarrow e \gamma$. This is enhanced by a single tan $\beta$ with respect to the $\varepsilon$ obtained with an external $m_{\mu}$ insertion, and has a larger numerical factor. For $\tan \beta \sim 1$, this is less than the expectation in the previous "maximal" pattern 
(see eq. (4.13)), due to the additional factors of $Y_{d}$. However, $\varepsilon \propto \tan ^{7} \beta$ grows rapidly with $\tan \beta$, and would exceed the current bound on $\mu \rightarrow e \gamma$ for $\tan \beta \gtrsim 10$.

Unlike the "maximal" pattern of section 4.1, the coefficients of quark bilinears are flavour changing for doublet and singlet up-type quarks, as well as the charged current. Since the $S_{0}$ and $\tilde{S}_{2}$ leptoquarks carry "down-type" flavour, they do not mediate flavour-changing interactions among down-type quarks at tree level. ${ }^{10}$ The induced quark bilinears are for $S_{0}$ are

$$
\begin{array}{ll}
{\left[K D_{d}^{2}\right]_{P S}\left(\bar{u}_{P} \gamma^{\mu} P_{L} d_{S}\right),} & {\left[K D_{d}^{2} K^{\dagger}\right]_{P S}\left(\bar{u}_{P} \gamma^{\mu} P_{L} u_{S}\right),} \\
{\left[D_{u} K D_{d}^{2} K^{\dagger} D_{u}\right]^{p s}\left(\bar{u}_{p} \gamma^{\mu} P_{R} u_{s}\right),} & y_{d, S}^{2}\left(\bar{d}_{S} \gamma^{\mu} P_{L} d_{S}\right)\left(S_{0}\right),
\end{array}
$$

and for $\tilde{S}_{2}$ :

$$
y_{d, s}^{2}\left(\bar{d}_{s} \gamma^{\mu} P_{R} d_{s}\right)\left(\tilde{S}_{2}\right)
$$

where $p, s(P, S)$ are singlet (doublet) generation labels, not to be summed over. Flavour changing $V \pm A$ bilinears among the up-type quarks, are suppressed by $y_{f}^{2}$ for doublets and $y_{f}^{4}$ for singlets, so neutral pseudoscalar meson decays induced in this pattern with be undetectable: $B$ and $K$ decays do not arise, and $D \rightarrow \mu \bar{e}$ is small. Any quark bilinear is suppressed by $y_{f}^{2}$ or $y_{f}^{4}$, as compared to the (undetected) interactions of the Higgs, which couples to quark bilinears with a single power of $y_{f}$.

Pseudoscalar operators are generated by the two chiral couplings of $S_{0}$, with quark bilinears and coefficients:

$$
y_{d, P}^{2} K_{r P}^{*} y_{u, r}\left(\bar{d}_{P} P_{R} u_{r}\right), \quad K_{s P} y_{d, s}^{2} K_{r s}^{*} y_{u, r}\left(\bar{u}_{P} P_{R} u_{r}\right)
$$

The flavour diagonal pseudoscalars are undetectable compared to the pseudoscalar couplings of the Higgs $\propto y_{f}$. From eq. (4.15) and eq. (4.16), we see that $\lambda_{L S_{0}} \lambda_{R S_{0}} \sim$ $y_{e, i} y_{u, r} \lambda_{L S_{0}}^{2}$, so the contribution of the pseudoscalar operator to pseudoscalar meson decays is smaller, by the factor $y_{u, i}$, than that of the $V-A$ operator. Therefore in table 3 , we do not estimate rates for pseudoscalar meson decays in this pattern.

Some estimates for $\varepsilon \mathrm{s}$ can be found in table 3. Since this ansatz does not induce tree level FCNC among down-type quarks (no $B_{s} \rightarrow \mu \bar{\mu}, K_{L} \rightarrow \mu^{ \pm} e^{\mp}$ ), the most sensitive rare decay is $\mu \rightarrow e \gamma$.

Leptoquark decay to a charged lepton $(e, \mu)$ and a $t$ or $b$ is an interesting search channel for this pattern at hadron colliders (similarly to the "maximal" pattern of couplings discussed in section 4.1). For third generation leptoquarks, the couplings $\lambda_{L S_{0}}, \lambda_{L \tilde{S}_{2}} \gtrsim 10^{-8}$ for $\tan \beta \sim 1$, so the leptoquarks would decay within a few centimetres of the production point. However, a third generation $S_{0}$ with only the coupling $\lambda_{R S_{0}}$, could appear as a track in the detector, since the largest $\lambda \lesssim y_{\mu} y_{\tau}^{2} \delta y_{b} \tan ^{4} \beta \sim 10^{-10} \tan ^{4} \beta$. Lower generation leptoquarks have very small $\lambda \mathrm{s}$, potentially allowing them to hadronise and escape the detector. However, we imagine that in a more realistic model, there would be intergeneration mixing among leptoquarks, which could allow faster decays.

\footnotetext{
${ }^{10}$ If the possibility of generation-mixing via the leptoquark mass matrix was included — as happens for instance for squarks - this would no longer be the case.
} 


\section{Leptoquarks with quark and lepton flavour}

The final possibility that we consider is to attribute both quark and lepton flavour to the leptoquarks. There are numerous possibilities. To avoid listing them all, we require, as discussed prior to section 4.1, that the $\lambda$ matrices be proportional to quark Yukawa matrices, and that hypercharge survive as a global symmetry in the presence of the leptoquark couplings $\lambda$ (see section 4.2). In practice, the second condition requires the sum of the hypercharges of the flavour spaces in which the leptoquark lives should be the hypercharge of the leptoquark. This allows the following $\lambda \mathrm{s}$ :

$$
\begin{aligned}
S_{0} \lambda_{\mathbf{L} \mathbf{S}_{\mathbf{0}}} \bar{\ell} i \tau_{2} q^{c} & \rightarrow S_{0}^{j q}\left[\mathbf{Y}_{\mathbf{e}}^{*}\right]^{I j}\left[\mathbf{Y}_{\mathbf{u}}^{*}\right]^{P q} \bar{\ell}_{I} i \tau_{2} q_{P}^{c} \\
S_{0} \lambda_{\mathbf{R S}_{\mathbf{0}}} \bar{e} u^{c} & \rightarrow S_{0}^{j q}\left[\widetilde{\mathbf{W}}_{\mathbf{e}}\right]^{i j}\left[\widetilde{\mathbf{W}}_{\mathbf{u}}\right]^{p q} \bar{e}_{i} u_{p}^{c} \\
\tilde{S}_{0} \tilde{\lambda}_{\mathbf{R}_{\mathbf{S}_{\mathbf{0}}}} \bar{e} d^{c} & \rightarrow \tilde{S}_{0}^{j q}\left[\widetilde{\mathbf{W}}_{\mathbf{e}}\right]^{i j}\left[\widetilde{\mathbf{W}}_{\mathbf{d}}\right]^{p q} \bar{e}_{i} d_{p}^{c} \\
\lambda_{\mathbf{L} \mathbf{S}_{\mathbf{2}}} \bar{\ell} u S_{2} & \rightarrow\left[\mathbf{Y}_{\mathbf{e}}^{*}\right]^{I j}\left[\mathbf{Y}_{\mathbf{u}}^{*}\right]^{P q} \bar{\ell}_{I} u_{q} S_{2}^{j P} \\
\lambda_{\mathbf{R S}_{\mathbf{2}}} \bar{e}\left[i \tau_{2} q\right]^{T} S_{2} & \rightarrow\left[\widetilde{\mathbf{W}}_{\mathbf{e}}\right]^{j i}\left[\mathbf{W}_{\mathbf{u}}\right]^{Q P} \bar{e}_{i}\left[q_{Q} i \tau_{2}\right]^{T} S_{2}^{j P} \\
\tilde{\lambda}_{\mathbf{L}_{\mathbf{2}}} \bar{\ell} d \tilde{S}_{2} & \rightarrow\left[\mathbf{W}_{\mathbf{e}}\right]^{J I}\left[\widetilde{\mathbf{W}}_{\mathbf{d}}\right]^{q p} \bar{\ell}_{I} d_{q} \tilde{S}_{2}^{J p}
\end{aligned}
$$

Giving lepton and quark flavour to the leptoquarks will lead to a multiplicity of leptoquarks: each of the five possible singlet and doublet leptoquarks will come in 3 colours and 9 flavours. This can be consistent with precision electroweak data, if these particles obtain mass other than by interacting with the Higgs, as discussed at the end of section 2 .

The quark and lepton indices of the $\lambda$ s are unrelated in this pattern, so the quark and lepton bilinears, and their coefficients, can be studied separately. In the mass eigenstate bases of the quarks, the bilinears are suppressed by two or four powers of Yukawa eigenvalues, and FCNC arise via CKM. The $V \pm A$ bilinears mediated by the nine types of $S_{0}$ are $\left[D_{u}^{2} K\right]_{P S}\left(\bar{u}_{P} \gamma^{\mu} P_{L} d_{S}\right), \quad\left[K^{\dagger} D_{u}^{2} K\right]_{P S}\left(\bar{d}_{P} \gamma^{\mu} P_{L} d_{S}\right), \quad y_{u, S}^{2}\left(\bar{u}_{S} \gamma^{\mu} P_{L} u_{S}\right), \quad y_{u, p}^{4}\left(\bar{u}_{p} \gamma^{\mu} P_{R} u_{p}\right)$,

where the $\left(\bar{d} \gamma P_{L} d\right)$ bilinear combines with neutrinos, and the others with two charged leptons or a charged current as required (see table 1). The doublet $S_{2}$ gives

$$
\left[K^{\dagger} D_{u}^{4} K\right]_{P T}\left(\bar{d}_{P} \gamma^{\mu} P_{L} d_{T}\right), \quad y_{u, p}^{4}\left(\bar{u}_{p} \gamma^{\mu} P_{L} u_{p}\right), \quad y_{u, p}^{2}\left(\bar{u}_{p} \gamma^{\mu} P_{R} u_{p}\right),
$$

and both $S_{0}$ and $S_{2}$ can mediate pseudoscalar operators, with coefficients

$$
K_{s P}^{*} y_{u, s}^{3}\left(\bar{d}_{P} P_{R} u_{s}\right), \quad y_{u, r}^{3}\left(\bar{u}_{r} P_{R} u_{r}\right)
$$

Finally the leptoquarks interacting with singlet $d \mathrm{~s}\left(\tilde{S}_{2}, \tilde{S}_{0}\right)$ induce:

$$
y_{d, s}^{4}\left(\bar{d}_{s} \gamma^{\mu} P_{R} d_{s}\right) .
$$

Lepton flavour is conserved for the $\lambda$ s of eq. (5.1) to eq. (5.6), because the leptonic part of the $\lambda \mathrm{s}$ is constructed only with $Y_{e}$. At tree level, this pattern therefore generates $V \pm A$ four fermion operators that arise in the SM, with coefficients $\propto y_{f}^{4}, y_{f}^{6}$ or $y_{f}^{8}$. These 
can be compared to four fermion interactions induced by the Higgs boson, which have a coefficient $\propto y_{f}^{2}$ and are unobserved. The most sensitive process would be $B_{s} \rightarrow \mu \bar{\mu}$ (induced by a $V \pm A$ operator, because the leptoquarks of eq. (2.5) do not generate a pseudoscalar operator $\left(\bar{d} P_{R} d\right)\left(\bar{e} P_{L} e\right)$, see table 1$)$. However, the $B_{s} \rightarrow \mu \bar{\mu}$ amplitude is suppressed by an extra $y_{\mu}^{2}$ with respect to the SM, so large $\tan \beta$ would be required to detect it. The pseudoscalar operators are also sufficiently suppressed.

Leptoquark-quark loops can induce flavour diagonal lepton dipole operators, such as $(g-2)_{\mu}[48,49]$. However, it is easy to see that the contribution to $(g-2)_{\mu}$ is always negligeable. The one loop SM electroweak contribution $\simeq G_{F} m_{\mu}^{2} /\left(8 \pi^{2}\right)$ is of order the experimental uncertainty, and we can guesstimate that the leptoquark loops $\lesssim N_{c} \lambda^{2} m_{\mu}^{2} /\left(8 \pi^{2} m_{S}^{2}\right)$. Since the $\lambda_{\mathrm{s}}$ which couple to muons are proportional to $y_{\mu}$, this is very small.

Another potentially interesting process is $b \rightarrow s \gamma$; with a $\tau$ or $\nu_{\tau}$ in the loop. From eq. (2.11), we obtain (for $S_{0}$ and $\nu_{\tau}$ in the loop)

$$
\varepsilon \sim \frac{1}{6} \frac{1}{96 \pi^{2}} y_{\tau}^{2} y_{t}^{2} K_{t b} K_{t s}
$$

Assuming that the leptoquarks can contribute at most $\sim 30 \%$ of the SM $b \rightarrow s \gamma$ rate, we estimate $\varepsilon^{s b} \lesssim 2 \times 10^{-4}$. As can be seen from table $4, b \rightarrow s \gamma$ is less sensitive than $B_{s} \rightarrow \mu \bar{\mu}$, because the loop suppression more than compensates for the larger $\tau$ Yukawas.

To obtain lepton flavour violation in this pattern, we can introduce the lepton number conserving spurion associated to the majorana mass matrix:

$$
\widetilde{W}_{\nu} \equiv \frac{1}{m_{\mathrm{atm}}^{2}}\left[m_{\nu}\right]\left[m_{\nu}\right]^{\dagger}=U D_{\nu}^{2} U^{\dagger}
$$

where $U$ is the leptonic mixing matrix, we assume a normal hierarchy for neutrino masses so $D_{\nu}^{2}=\operatorname{diag}\left\{0, \delta^{2}, 1\right\}$, and we neglected the lightest neutrino mass.

This additional spurion could multiply various lepton doublet indices appearing in the construction of the $\lambda_{\mathrm{s}}$. For instance, if we maintain the lepton doublets in the phenomenologically relevant charged lepton mass basis, we can nonetheless perform the replacement: ${ }^{11}$

$$
\widetilde{W}_{e} \rightarrow Y_{e}^{\dagger} \widetilde{W}_{\nu} Y_{e}
$$

in eq. (5.1) to eq. (5.6) above, which allows the LFV bilinears

$$
\begin{aligned}
{\left[D_{e} U D_{\nu}^{2} U^{\dagger} D_{e}^{2} U D_{\nu}^{2} U^{\dagger} D_{e}\right]^{i j}\left(\bar{e}_{i} \gamma^{\rho} P_{R} e_{j}\right) } & \simeq y_{e, i} U_{i 3} \frac{y_{\tau}^{2}}{2} U_{j 3} y_{e, j}\left(\bar{e}_{i} \gamma^{\rho} P_{R} e_{j}\right) \\
{\left[D_{e}^{2} U D_{\nu}^{2} U^{\dagger} D_{e}\right]^{I j}\left(\bar{e}_{I} P_{R} e_{j}\right) } & \simeq y_{e, I}^{2} U_{I 3} U_{j 3} y_{e, j}\left(\bar{e}_{I} P_{R} e_{j}\right)
\end{aligned}
$$

The last approximation assumes that $U_{e 3}=\sin \theta_{13} \gg \delta^{2} \simeq .03$. This allows the $\varepsilon$ s listed in the last colomn of table 4 , where we approximate $U_{\mu 3} \simeq U_{\tau 3} \simeq 1$.

In this pattern which allows for lepton flavour violation, the most sensitive rare decay would be $\tau \rightarrow \mu \gamma$, where the predicted amplitude (due to $S_{2}$ exchange) becomes of order of the current bound for $\tan \beta \sim 80$. As in previous sections, the loop contribution to

\footnotetext{
${ }^{11}$ The replacement $W_{e} \rightarrow \widetilde{W}_{\nu} W_{e}$ in eq. (5.6) is also possible, but for simplicity we do not consider it.
} 


\begin{tabular}{|c|c|c|c|}
\hline process & $\varepsilon<$ & $S$ with $\mathrm{Q}$ and $\mathrm{L}$ flav & $S$ with Q and L flav, and LFV \\
\hline$K^{+} \rightarrow \pi^{+} \nu \bar{\nu}$ & $9 \times 10^{-6}$ & $\frac{1}{6} y_{\tau}^{2} K_{t s} y_{t}^{2} K_{t d} \sim 5 \times 10^{-9} t_{\beta}^{2}$ & $\frac{1}{6} y_{\tau}^{2} K_{t s} y_{t}^{2} K_{t d} \sim 5 \times 10^{-9} t_{\beta}^{2}$ \\
\hline$K_{L} \rightarrow \mu^{ \pm} e^{\mp}$ & $3 \times 10^{-7}$ & & $\begin{array}{r}\frac{1}{12} y_{\mu} y_{\tau}^{2} s_{13} y_{e} y_{t}^{4} K_{t s} K_{t d} \\
\sim \frac{s_{13}}{.1} 5 \times 10^{-19} t_{\beta}^{4}\end{array}$ \\
\hline$B_{s} \rightarrow \mu^{ \pm} \mu^{\mp}$ & $7 \times 10^{-5}$ & $\frac{1}{6} y_{\mu}^{4} y_{t}^{4} K_{t s} K_{t b} \sim 10^{-15} t_{\beta}^{4}$ & $\frac{1}{6} y_{\mu}^{2} y_{\tau}^{2} y_{t}^{4} K_{t s} K_{t b} \sim 2 \times 10^{-13} t_{\beta}^{4}$ \\
\hline$B^{+} \rightarrow K^{+} \tau^{ \pm} \mu^{\mp}$ & $2 \times 10^{-3}$ & & $\frac{1}{12} y_{\mu} y_{\tau}^{3} y_{t}^{4} K_{t s} K_{t b} \sim 10^{-12} t_{\beta}^{4}$ \\
\hline$b \rightarrow s \gamma$ & $\sim 2 \times 10^{-4}$ & $\begin{array}{l}\frac{1}{576 \pi^{2}} y_{\tau}^{2} y_{t}^{2} K_{t b} K_{t s} \\
\quad \sim 7 \times 10^{-10} t_{\beta}^{2}\end{array}$ & $\frac{1}{576 \pi^{2}} y_{\tau}^{2} y_{t}^{2} K_{t b} K_{t s} \sim 7 \times 10^{-10} t_{\beta}^{2}$ \\
\hline$\mu N \rightarrow e N^{\prime}$ & $8 \times 10^{-7}$ & & $\frac{1}{64 \pi^{2}} y_{\mu} y_{\tau}^{2} s_{13} y_{e} y_{t}^{2} \sim 2 \frac{s_{13}}{.1} 10^{-17} t_{\beta}^{4}$ \\
\hline$\mu \rightarrow e \gamma$ & $\sim 10^{-6}$ & & $\frac{1}{64 \pi} y_{\mu} y_{\tau}^{2} s_{13} y_{e} y_{t}^{2} \sim \frac{s_{13}}{.1} 10^{-16} t_{\beta}^{4}$ \\
\hline$\tau \rightarrow \mu \gamma$ & $\sim 10^{-4}$ & & $\frac{1}{64 \pi} y_{\mu} y_{\tau}^{3} y_{t}^{2} \sim 2 \times 10^{-12} t_{\beta}^{4}$ \\
\hline
\end{tabular}

Table 4. The largest expected amplitudes, induced by any scalar leptoquark of mass $\sim 300 \mathrm{GeV}$, with the flavoured coupling $\lambda^{\mathrm{LQ}}$ arising when the leptoquark carries quark and lepton flavour. The second colomn is the bound on $\varepsilon$ (defined in eq. (2.7)) for the process in the first colomn, the following colomns are the expectations respectively with and without lepton flavour violation via the neutrino mass matrix.

$\mu N \rightarrow e N^{\prime}$, of a third generation $S_{0}$ (with a $b$ in the loop) or $S_{2}$ (with $t$ or $b$ in the loop), which is listed in table 4 , dominates over the tree contribution of an $S_{2}$ to $\mu N \rightarrow e N^{\prime}$ :

$$
\left.\varepsilon^{e \mu d d} \simeq \frac{1}{6} y_{\mu} y_{\tau}^{2} s_{13} y_{e} K_{t d}^{2} y_{t}^{4} \sim 2 \frac{s_{13}}{.1} 10^{-19} t_{\beta}^{4} \quad \text { (tree, } S_{2}\right)
$$

The estimates in table 4 show that leptoquarks with quark and lepton flavour remains difficult to detect in rare decays, even with the addition of lepton flavour violation. However, if intergeneration mixing among the leptoquarks was allowed, as could be expected in a realistic model of leptoquark masses, the rare decay rates could be enhanced.

Leptoquarks with such a pattern of couplings could have interesting signatures at colliders. In the absence of intergeneration mixing, the lower generation leptoquarks could hadronize and travel in the detector before they decay. However the third generation 
leptoquarks decay promptly to $t \mathrm{~s}$ or $b \mathrm{~s}$ and $\tau \mathrm{s}$, and in the case of $S_{0}$ and $S_{2}$ leptoquarks of second lepton and third quark generation, the decay to a $\mu$ and a third generation quark also takes place within a few centimetres of the production point for $\tan \beta \sim 1$.

\section{Summary and discussion}

Data from rare decay searches and collider experiments implies that leptoquarks with $m_{S} \lesssim \mathrm{TeV}$ should not have $\mathcal{O}(1)$ couplings to leptons and quarks of arbitrary flavour. This can be quantified as constraints on a dimensionless $\varepsilon$ coefficient of dimension six operators, as defined in equations (2.7) and (2.9). Indeed, it is well known that New Physics at the electrweak scale should have its flavoured interactions patterned on those of the Standard Model. For several New Physics scenarios, such as Supersymmetry, this can be elegantly obtained by imposing Minimal Flavour Violation (MFV). However, since the Standard Model does not provide a template interaction (which could serve as a "spurion") linking leptons and quarks, it is not obvious how to apply the elegant formulation of MFV of d'Ambrosio et al. [26] to leptoquarks. Phrased another way, the leptoquark coupling matrix $\lambda$ has one lepton generation index and one quark generation index; how can this be constructed from the SM mass matrices, which have two lepton, or two quark indices? In this paper, we explore three ways to construct the leptoquark-quark-lepton couplings $\lambda$ out of the observed mass matrices. For simplicity, to reduce the permutations, we consider only electroweak singlet and doublet scalar leptoquarks.

1. In section 3, the leptoquarks have neither lepton nor quark flavour, but a new "spurion", or flavour structure, is introduced. It is a unit matrix, because this is the most minimal of structures, and it connects the mass eigenstate basis of singlet $u$-type quarks, to the mass eigenstate basis of charged leptons. This ensures that the new spurion does not introduce any new bases in the vector spaces of flavour, and avoids the stringent bounds from $B$ and $K$ decays. The leptoquark couplings to other types of quark or lepton can be obtained by multiplying the unit matrix by Yukawa matrices, as given in eq. (3.3) and eq. (3.4).

This pattern of leptoquark couplings has the interesting feature of favouring interactions between leptons and quarks of the same generation. This is an "intuitive" expectation for leptoquark couplings, which could be interpreted as a leptoquark signature. It is to avoid the strict constraint from $K_{L} \rightarrow \mu^{ \pm} e^{\mp}$ that the unit matrix connects singlet $u$-type quarks to leptons.

The (tree level) four fermion operator coefficients generated in this pattern are given in eq. (3.5) to eq. (3.8). Generation non-diagonal quark-lepton couplings can arise due to CKM, in the presence of $d$-type quarks. Since the unit matrix connects singlet $u$ s to the leptons of the same generation, the $\lambda$ couplings of leptons to $d$-type quarks are proportional to quark Yukawa matrices. Leptoquarks therefore have stronger couplings to $b$ quarks than $s$ quarks; $B \rightarrow e \nu_{\tau}, \tau \nu_{e}$ are among the most sensitive low energy processes, followed by $K^{+} \rightarrow \pi^{+} \nu_{\tau} \bar{\nu}_{\tau}$. In the table 2 are listed the $\varepsilon$ factors for various rare processes which could be sensitive to this pattern of leptoquark couplings. 
2. The second prospect, explored in section 4 , is to attribute quark generation number to the leptoquark. The $\lambda$ couplings thus have one quark flavour index for the leptoquark and one for the quark, so can be proportional to a quark Yukawa matrix. The single lepton index of $\lambda$ can be obtained following an idea of Nikolidakis and Smith [23], discussed around eq. (4.1), which combines the antisymmetrric $\epsilon_{I J K}$ with the majorana neutrino mass matrix $\left[m_{\nu}\right]_{I J}$.

In this approach, we must choose the quark flavour space in which to place the leptoquark. The first possibility which we study, in section 4.1, is to choose the largest Yukawa matrix interacting with the quark at the vertex, which fixes the flavour space for the leptoquark. However, a more "consistent" approach, studied in section 4.2 , might be to place the leptoquark in the flavour space of quarks who have the same hypercharge.

Leptoquark couplings $\lambda$ constructed according to this pattern do not relate the quark to lepton generation indices. The interaction with quarks is proportional to (one or two powers of) quark Yukawa matrices, so they are hierarchical, with flavour changing neutral currents suppressed as in the usual Minimal Flavour Violation. This can be seen from the coefficients of quark bilinears, which contribute to the four fermion operators induced by the leptoquarks, and which are given in eq. (4.10), eq. (4.19) and eq. (4.20). The two cases we consider differ in that the tree level FCNC are among $d$-type quarks in section 4.1, and among $u$-type quarks in section 4.2. All three generations of leptoquark have similar interactions to es and $\mu \mathrm{s}$, with some suppression to $\tau \mathrm{s}$ (see eq. (4.12)), due to the democratic structure of the majorana neutrino mass matrix, which provides the flavour violation. Due to the hierarchy in couplings to quarks, the most sensitive decay for these patterns would be $\mu \rightarrow e \gamma$ with a $t$ or $b$ in the loop. In the table 3 the $\varepsilon$ factors for various other processes are listed.

3. Finally in section 5 we consider leptoquarks carying lepton and quark generation indices. This implies a large number of leptoquarks (3 colours $\times 3 \times 3$ generations), and very hierarchical couplings $\lambda \propto y_{f}^{2}, y_{f}^{3}$ or $y_{f}^{4}$, where $y_{f}$ is a Yukawa coupling. Table 4 lists some estimates for rare processes in two cases: quark generation change via CKM, with or without lepton flavour violation via the lepton mixing matrix. Processes such as $\tau \rightarrow \mu \gamma$ could be sensitive to third generation leptoquarks, particularily for large $\tan \beta$. If produced at hadron colliders, such third generation leptoquarks would decay to $t$ or $b$ and $\tau$, or possibly $\mu$. However, realistically, leptoquarks of different generations could be expected to mix, which could significantly modify the expectations, due to the steep hierarchy of couplings.

In the tables 2, 3 and 4 are listed the $\varepsilon$ factors, for selected rare processes, which arise for the patterns of leptoquark couplings considered in this paper. Although the expectation for the various patterns differ, various prospects can be anticipated:

- For $\tan \beta=1$, the expectations are well below the experimental bounds. So electroweak-scale leptoquarks are possible, and could be produced via their gauge 
interactions at hadron colliders. The particular collider signatures of each pattern are briefly discussed at then ends of sections 3.1, 4.1, 4.2 and 5. Several of the leptoquarks we consider decay preferentially to third generation fermions, so leptoquark searches at the Tevatron and the LHC for final states containing tops and/or taus would be interesting.

- Lepton flavour violating observables, such as $\tau \rightarrow \mu \gamma, \mu \rightarrow e \gamma$, and $\mu N \rightarrow e N^{\prime}$, are sensitive probes of (third generation) leptoquarks, because the leptoquarks can contibute via loops with third generation couplings, and the experimental bounds on these clean processes are good.

- Finally, a question for models of $\lambda$ couplings, is "which meson decays are most sensitive to leptoquarks?" Putting aside the D decays, because the experimental bounds are less restrictive, this amounts to comparing the predicted branching ratios for $B$ and $K$ decays to the current bounds. The latter can be several orders of magnitude more stringent for $K \mathrm{~s}$ than for $B \mathrm{~s}$. One can roughly estimate that $K$ decays may be slightly more sensitive, when $\lambda \propto y_{f}$, as can arise in section 3 , or if $\lambda \propto \sqrt{y_{f} y_{f}^{\prime}}$. The patterns discussed in this paper did not give a square root, but it is expected in the Cheng-Sher ansatz [50], and can arise in various types of models such as [8] (expectations with this ansatz also are discussed in [14]). However, if $\lambda \propto\left(y_{f} y_{f}^{\prime}\right)^{n}$, for $n \geq 1$, as arises in most of the patterns we consider here, then $B$ decays are a better place to look for leptoquarks.

\section{Acknowledgments}

We thank Gino Isidori for contributions, and SD thanks Uli Haisch for a seminar invitation. This work was partially supported by the EU Contract No. MRTN-CT-2006-035482, FLAVIAnet.

Open Access. This article is distributed under the terms of the Creative Commons Attribution Noncommercial License which permits any noncommercial use, distribution, and reproduction in any medium, provided the original author(s) and source are credited.

\section{References}

[1] S. Davidson, D.C. Bailey and B.A. Campbell, Model independent constraints on leptoquarks from rare processes, Z. Phys. C 61 (1994) 613 [hep-ph/9309310] [SPIRES].

[2] J.L. Hewett and T.G. Rizzo, Much ado about leptoquarks: a comprehensive analysis, Phys. Rev. D 56 (1997) 5709 [hep-ph/9703337] [SPIRES].

[3] P. Langacker, Grand Unified Theories and proton decay, Phys. Rept. 72 (1981) 185 [SPIRES].

[4] E. Farhi and L. Susskind, Technicolor, Phys. Rept. 74 (1981) 277 [SPIRES].

[5] R. Barbier et al., R-parity violating supersymmetry, Phys. Rept. 420 (2005) 1 [hep-ph/0406039] [SPIRES]. 
[6] S.S. Gershtein, A.A. Likhoded and A.I. Onishchenko, TeV-scale leptoquarks from GUTs/string/M-theory unification, Phys. Rept. 320 (1999) 159 [SPIRES].

[7] I. Dorsner and P. Fileviez Perez, Unification without supersymmetry: neutrino mass, proton decay and light leptoquarks, Nucl. Phys. B 723 (2005) 53 [hep-ph/0504276] [SPIRES].

[8] B. Gripaios, Composite leptoquarks at the LHC, JHEP 02 (2010) 045 [arXiv:0910.1789] [SPIRES].

[9] P. Fileviez Perez, T. Han, T. Li and M.J. Ramsey-Musolf, Leptoquarks and neutrino masses at the LHC, Nucl. Phys. B $\mathbf{8 1 9}$ (2009) 139 [arXiv: 0810.4138] [SPIRES].

[10] D. Aristizabal Sierra, M. Hirsch and S.G. Kovalenko, Leptoquarks: neutrino masses and accelerator phenomenology, Phys. Rev. D 77 (2008) 055011 [arXiv:0710.5699] [SPIRES].

[11] P.Y. Popov, A.V. Povarov and A.D. Smirnov, Fermionic decays of scalar leptoquarks and scalar gluons in the minimal four color symmetry model, Mod. Phys. Lett. A 20 (2005) 3003 [hep-ph/0511149] [SPIRES].

[12] M. Raidal et al., Flavour physics of leptons and dipole moments, Eur. Phys. J. C 57 (2008) 13 [arXiv:0801.1826] [SPIRES].

[13] J.P. Saha, B. Misra and A. Kundu, Constraining scalar leptoquarks from the $K$ and $B$ sectors, Phys. Rev. D 81 (2010) 095011 [arXiv: 1003.1384] [SPIRES].

[14] M. Carpentier and S. Davidson, Constraints on two-lepton, two quark operators, arXiv: 1008.0280 [SPIRES].

[15] D0 collaboration, V.M. Abazov et al., Search for pair production of first-generation leptoquarks in p $\bar{p}$ collisions at $\sqrt{s}=1.96 \mathrm{TeV}$, Phys. Lett. B 681 (2009) 224 [arXiv: 0907.1048] [SPIRES].

[16] D0 collaboration, V.M. Abazov et al., Search for pair production of second generation scalar leptoquarks, Phys. Lett. B 671 (2009) 224 [arXiv:0808.4023] [SPIRES].

[17] D0 collaboration, V.M. Abazov et al., Search for third generation scalar leptoquarks decaying into $\tau$ b, Phys. Rev. Lett. 101 (2008) 241802 [arXiv:0806.3527] [SPIRES].

[18] D0 collaboration, V.M. Abazov et al., Search for scalar bottom quarks and third-generation

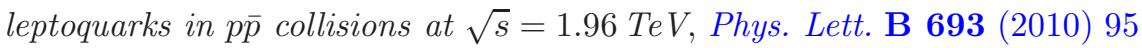
[arXiv: 1005.2222] [SPIRES].

[19] M. Krämer, T. Plehn, M. Spira and P.M. Zerwas, Pair production of scalar leptoquarks at the LHC, Phys. Rev. D 71 (2005) 057503 [hep-ph/0411038] [SPIRES].

[20] O.J.P. Eboli, R. Zukanovich Funchal and T.L. Lungov, Signal and backgrounds for leptoquarks at the CERN LHC, Phys. Rev. D 57 (1998) 1715 [hep-ph/9709319] [SPIRES].

[21] A. Belyaev, C. Leroy, R. Mehdiyev and A. Pukhov, Leptoquark single and pair production at LHC with CalcHEP/CompHEP in the complete model, JHEP 09 (2005) 005 [hep-ph/0502067] [SPIRES].

[22] ATLAS collaboration, C. Boulahouache, Prospects for early discoveries in final states with dileptons and jets: LRSM and leptoquarks, AIP Conf. Proc. 1078 (2009) 584 [SPIRES].

[23] E. Nikolidakis and C. Smith, Minimal Flavor Violation, seesaw and R-parity, Phys. Rev. D 77 (2008) 015021 [arXiv:0710.3129] [SPIRES]. 
[24] A.J. Buras, P. Gambino, M. Gorbahn, S. Jager and L. Silvestrini, Universal unitarity triangle and physics beyond the standard model, Phys. Lett. B 500 (2001) 161 [hep-ph/0007085] [SPIRES].

[25] R.S. Chivukula and H. Georgi, Composite technicolor standard model, Phys. Lett. B 188 (1987) 99 [SPIRES].

[26] G. D'Ambrosio, G.F. Giudice, G. Isidori and A. Strumia, Minimal Flavour Violation: an effective field theory approach, Nucl. Phys. B 645 (2002) 155 [hep-ph/0207036] [SPIRES].

[27] T. Feldmann and T. Mannel, Minimal Flavour Violation and beyond, JHEP 02 (2007) 067 [hep-ph/0611095] [SPIRES].

[28] V. Cirigliano, B. Grinstein, G. Isidori and M.B. Wise, Minimal Flavor Violation in the lepton sector, Nucl. Phys. B 728 (2005) 121 [hep-ph/0507001] [SPIRES].

[29] S. Davidson and F. Palorini, Various definitions of Minimal Flavour Violation for leptons, Phys. Lett. B 642 (2006) 72 [hep-ph/0607329] [SPIRES].

[30] M.B. Gavela, T. Hambye, D. Hernandez and P. Hernández, Minimal flavour seesaw models, JHEP 09 (2009) 038 [arXiv:0906.1461] [SPIRES].

[31] B. Grinstein, V. Cirigliano, G. Isidori and M.B. Wise, Grand unification and the principle of Minimal Flavor Violation, Nucl. Phys. B 763 (2007) 35 [hep-ph/0608123] [SPIRES].

[32] W. Buchmüller, R. Ruckl and D. Wyler, Leptoquarks in lepton quark collisions, Phys. Lett. B 191 (1987) 442 [Erratum ibid. B 448 (1999) 320] [SPIRES].

[33] ZEUS collaboration, S. Chekanov et al., A search for resonance decays to lepton + jet at HERA and limits on leptoquarks, Phys. Rev. D 68 (2003) 052004 [hep-ex/0304008] [SPIRES].

[34] H1 collaboration, A. Aktas et al., Search for leptoquark bosons in e p collisions at HERA, Phys. Lett. B 629 (2005) 9 [hep-ex/0506044] [SPIRES].

[35] H1 and ZEUS collaborations, R. Ciesielski, Search for leptoquarks and contact interactions at HERA, PoS (EPS-HEP 2009) 269 [SPIRES].

[36] J.K. Mizukoshi, O.J.P. Eboli and M.C. Gonzalez-Garcia, Bounds on scalar leptoquarks from Z physics, Nucl. Phys. B 443 (1995) 20 [hep-ph/9411392] [SPIRES].

[37] G. Bhattacharyya, J.R. Ellis and K. Sridhar, Bounds on the masses and couplings of leptoquarks from leptonic partial widths of the Z, Phys. Lett. B 336 (1994) 100 [Erratum ibid. B 338 (1994) 522] [hep-ph/9406354] [SPIRES].

[38] L. Lavoura, General formulae for $f_{1} \rightarrow f_{2} \gamma$, Eur. Phys. J. C 29 (2003) 191 [hep-ph/0302221] [SPIRES].

[39] M. Hirsch, H.V. Klapdor-Kleingrothaus and S.G. Kovalenko, New low-energy leptoquark interactions, Phys. Lett. B 378 (1996) 17 [hep-ph/9602305] [SPIRES].

[40] E. Keith and E. Ma, Oblique $S$ and T parameters and leptoquark models of the HERA events, Phys. Rev. Lett. 79 (1997) 4318 [hep-ph/9707214] [SPIRES].

[41] P.H. Frampton and M. Harada, Constraints from precision electroweak data on leptoquarks and bileptons, Phys. Rev. D 58 (1998) 095013 [hep-ph/9711448] [SPIRES].

[42] A.D. Smirnov, Bounds on scalar leptoquark and scalar gluon masses from S, T, $U$ in the minimal four color symmetry model, Phys. Lett. B 531 (2002) 237 [hep-ph/0202229] [SPIRES]. 
[43] L. Lavoura and L.-F. Li, Mechanism for obtaining a negative T oblique parameter, Phys. Rev. D 48 (1993) 234 [SPIRES].

[44] Particle Data Group collaboration, C. Amsler et al., Review of particle physics, Phys. Lett. B 667 (2008) 1 [SPIRES].

[45] CKM fitter homepage, http://ckmfitter.in2p3.fr/.

[46] A. Lenz et al., Anatomy of new physics in B- $\bar{B}$ mixing, arXiv:1008.1593 [SPIRES].

[47] UTfit collaboration, M. Bona et al., An improved standard model prediction of $B R(B \rightarrow \tau \nu)$ and its implications for new physics, Phys. Lett. B 687 (2010) 61 [arXiv:0908.3470] [SPIRES].

[48] K.-M. Cheung, Muon anomalous magnetic moment and leptoquark solutions, Phys. Rev. D 64 (2001) 033001 [hep-ph/0102238] [SPIRES].

[49] U. Mahanta, Implications of BNL measurement of $\delta\left(a_{\mu}\right)$ on a class of scalar leptoquark interactions, Eur. Phys. J. C 21 (2001) 171 [Phys. Lett. B 515 (2001) 111] [hep-ph/0102176] [SPIRES].

[50] T.P. Cheng and M. Sher, Mass matrix ansatz and flavor nonconservation in models with multiple Higgs doublets, Phys. Rev. D 35 (1987) 3484 [SPIRES]. 